\title{
ABSTRACT
}

Objectives. The overall objective of this application is to develop novel technologies for non-invasive imaging of adoptive stem cell-based therapies with positron emission tomography (PET) that would be applicable to human patients. To achieve this objective, stem cells will be genetically labeled with a PETreporter gene and repetitively imaged to assess their distribution, migration, differentiation, and persistence using a radiolabeled reporter probe. This new imaging technology will be tested in adoptive progenitor cellbased therapy models in animals, including: delivery pro-apoptotic genes to tumors, and T-cell reconstitution for immunostimulatory therapy during allogeneic bone marrow progenitor cell transplantation.

Technical and Scientific Merits. Non-invasive whole body imaging would significantly aid in the development and clinical implementation of various adoptive progenitor cell-based therapies by providing the means for non-invasive monitoring of the fate of injected progenitor cells over a long period of observation. The proposed imaging approaches could help to address several questions related to stem cell migration and homing, their long-term viability, and their subsequent differentiation. The ability to image these processes non-invasively in 3D and repetitively over a long period of time is very important and will help the development and clinical application of various strategies to control and direct stem cell migration and differentiation.

Approach to accomplish the work. Stem cells will be genetically with a reporter gene which will allow for repetitive non-invasive "tracking" of the migration and localization of genetically labeled stem cells and their progeny. This is a radically new approach that is being developed for future human applications and should allow for a long term (many years) repetitive imaging of the fate of tissues that develop from the transplanted stem cells.

Why the approach is appropriate. The novel approach to stem cell imaging is proposed to circumvent the major limitation of in vitro radiolabeling - the eventual radiolabel decay. Stable transduction of stem cells in vitro would allow for the selection of high quality stem cells with optimal functional parameters of the transduced reporter systems. The use of a long-lived radioisotope ${ }^{124} \mathrm{I}$ to label a highly specific reporter gene probe will allow for ex vivo labeling of stem cells and their imaging immediately after injection and during the following next week. The use of short-lived radioisotopes (i.e., ${ }^{18} \mathrm{~F}$ ) to label highly specific reporter gene probes will allow repetitive PET imaging for the assessment of to stem cell migration, targeting, differentiation, and long-term viability of stem cell-derived tissues.

Qualifications of the research team and resources. An established research team of experts in various disciplines has been assembled at MD Anderson Cancer Center (MDACC) over the past two years including the PI, senior co-investigators and collaborators. The participants of this team are recognized internationally to be among the leaders in their corresponding fields of research and clinical medicine. The resources at MDACC are exceptionally well developed and have been recently reinforced by the installation of a microPET and microSPECT/CT cameras, and a 7T MRI system for high resolution animal imaging; and by integrating a synthetic chemistry core for the development and production of precursors for radiolabeling. 


\section{BACKGROUND AND SIGNIFICANCE.}

EX vivo radiolabeling and nuclear imaging. Until recently, immunostaining of whole body slices of small animals was the most straightforward, reliable, and traditional approach used for the assessment of localization and targeting of adoptively transferred cells, such as the T-cells [1]. However, the invasive nature of classical pathology precludes the repetitive monitoring of cellular trafficking in the same animal over time. The results obtained from multiple animals sacrificed at different time points may be flawed by individual variations and a large number of animals have to be studied to achieve statistical significance. Another major shortcoming of this method is that it can not be applied in clinical setting. A method for non-invasive and repetitive evaluation of trafficking, homing, tumor targeting, differentiation, and persistence, would significantly aid the development of progenitor cell -based adoptive therapy approaches and facilitate their wider implementation in clinic.

Marking the cells with radiolabeled tracers was first tested in the early 1970-s. $\left[{ }^{3} \mathrm{H}\right]$ and $\left[{ }^{14} \mathrm{C}\right]$-labeled uridine was used to assess lymphocyte trafficking in a GVHD model [2]. Other earlier studies used [ ${ }^{3} \mathrm{H}$ ]glycerol [3]. A similar method, uisng $\left[{ }^{3} \mathrm{H}\right]$-labeled glucose was applied to patients to compare the kinetics of lymphocytes in the normal and HIV-infected individuals [4]. However, the use of gamma-emitting isotopes limits such studies to cellular kinetic studies based on sequential blood sampling. Non-invasive imaging of lymphocyte trafficking dates back to early $70-\mathrm{s}$, when the first experiments were performed with ${ }^{51} \mathrm{Cr}$ and ${ }^{99 \mathrm{~m}}$ Tc extracorporal labeling of lymphocytes $[5,6]$. These methods were applied to different immune cells using various radioisotopes for labeling (e.g., $\left.{ }^{111} \mathrm{In},{ }^{67} \mathrm{Co},{ }^{64} \mathrm{Cu}\right)[5-9]$. $\left[{ }^{111} \mathrm{In}\right]$ in particular, found a wide clinical application in oncology as an imaging agent for monitoring of vaccination with tumor-infiltrating lymphocytes $[10,11]$.

Imaging techniques that involve ex vivo labeling of lymphocytes or other adoptively transferred cells have several limitations. A major limitation of this approach is a relatively low attainable level of radioactivity per cell when labeling cells with passively equilibrating radiotracers such as the $\left[{ }^{111}\right.$ In]oxime and $\left[{ }^{64} \mathrm{Cu}\right] \mathrm{PTSM}$. Significantly higher levels of radioactivity per cell could be obtained with tracers such as $\left[{ }^{18} \mathrm{~F}\right] \mathrm{FDG}$ that utilize facilitated transport and enzyme-amplified accumulation. However, both the $\left[{ }^{64} \mathrm{Cu}\right] P T S M$ and $\left[{ }^{18} \mathrm{~F}\right] \mathrm{FDG}$ have been shown to gradually efflux out of the labeled cells [9]. Progressive loss of radiolabel also occurs during the cell division in vivo. More stable labeling of lymphocytes was achieved with $\left[{ }^{125} I\right] I U d R$ which stably incorporates into DNA of labeled cells [12]. Although, successful non-invasive imaging with $\left[{ }^{131} I\right] I U d R$ and $\left[{ }^{124} I\right] I U d R$ of tumors has been demonstrated with SPECT and PET, respectively [14, 15], and could be applied to gamma camera and PET imaging of radiolabeled lymphocytes, such an approach has never been tested. Another shortcoming of the ex vivo radiolabeling approach is the limited period of monitoring which is due to radiolabel decay and/or biological clearance. The exposure of cells to higher doses of radioactivity during labeling is also limited by radiotoxicity.

Labeling with supermagnetic agents and MR imaging. Recent developments in MR imaging have enabled in vivo imaging at near microscopic resolution [15-17]. In order to visualize and track stem and progenitor cells by MR imaging, it is necessary to tag cells magnetically. Conventional magnetic cell labeling techniques rely on surface attachment of magnetic beads ranging in size from several hundred nanometers to micrometers. Although these methods are efficient for in vitro cell separation, cell surface labeling is generally not suitable for in vivo use because of the rapid reticuloendothelial recognition and clearance of cells thus labeled. Alternatively, lymphocytes and other cells have been labeled with small monocrystalline nanoparticles ranging in sizes from 10 to $40 \mathrm{~nm}$ [18-24] using fluid-phase or receptor-mediated endocytosis. Unfortunately, the labeling efficiency is generally low, particularly in differentiated and nondividing cells.

Tat protein-derived peptide sequences have recently been used as an efficient way of internalizing a number of marker proteins into cells [25-27]. Lewin et al. (2000) demonstrated that hematopoietic CD34 ${ }^{+}$and neural progenitor cells (C17.2) can be very efficiently tagged with a novel triple-label (magnetic, fluorescent, isotope) superparamagnetic nanoparticle, termed CLIO-Tat [28]. They furthermore demonstrate that the labeled cells retain their capability for differentiation, can be visualized by high-resolution MR imaging, and can be retrieved from excised tissues and bone marrow using magnetic sorting techniques. However, the potential for a long term imaging (months or years) using in vitro labeled progenitor cells is limited due to eventual biological clearance of the magnetic label.

Genetic labeling of cells for adoptive therapies. Stable genetic labeling of adoptively transferred cells, such as lymphocytes, with various reporter genes has been used to circumvent the temporal limitations of in vitro radiolabeling or magnetic labeling of cells. A long-term circulation of the infused EBV specific cytotoxic 
donor-derived T cells in patient treated for post-BMT EBV-LPD was demonstrated by retrovirally transducing T cells with neomycin resistance or LNGFR and HSV-TK genes which were detectable in the peripheral blood samples from the patients by PCR or FACS analysis [29-31]. However, this method alone does not allow for the assessment spatial distribution of adoptively transferred cells. In this application, we propose to assess the non-invasive imaging of spatial and temporal dynamics of progenitor cells after their administration can be done by labeling the cells with PET-reporter genes which would be expressed in a constitutive and/or differentiation-specific manner.

\section{Reporter Gene Imaging Approaches.}

Bioluminescence Reporter Gene Imaging. Genetic labeling of lymphocytes with the luciferease reporter gene and non-invasive bioluminescence imaging (BLI) has been reported [34]. Although, the BLI of luciferase expressing CTL allows assessments of their distribution throughout the organism, it is semi-quantitative at best, because the signal intensity largely depends upon thickness and variable optical characteristics of tissues (see paper in Appendix I). Because of this shortcomming, the BLI of luciferase gene (Luc) expression has a limited applicability in clinic.

PET-Reporter Gene Imaging. Our group was the first to describe in 1995 the PET-reporter gene imaging paradigm [35]. This paradigm involves administering a radiolabeled probe which is selectively bound or metabolized (e.g., phosphorylated) and trapped by interaction with the gene product (e.g., an enzyme) in the reporter gene transduced cell. In this manner the bound or metabolized (e.g., phosphorylated) probe accumulates selectively in transduced tissue, and the level of probe accumulation is proportional to the level of gene product being expressed. It may be useful to consider reporter imaging paradigm as an enzymatic radiotracer assay that reflects reporter gene expression. Viewed from this perspective, reporter gene imaging is similar to imaging hexokinase activity with fluorodeoxyglucose (FDG). A recent review by Gambhir et al. (2000) provides a good overview of the founding principles of non-invasive reporter gene imaging [36].

PET imaging of herpesviral thymidine kinase (HSV1-tk) is a good example of a "reporter gene" for several reasons. HSV1-tk has been extensively studied; it is essentially non-toxic in humans and is currently being used in clinical gene therapy protocols as a "susceptibility" gene for treatment of cancer (in combination with Ganciclovir, GCV) [37]. A gene product of HSV1-tk is an enzyme, HSV1 thymidine kinase (HSV1-TK). Several group have demonstrated that HSV1-tk can be used as a "reporter gene" as well as a "therapeutic gene" [35, 36]. This represents an ideal situation where the "therapeutic" and "reporter" genes are the same. In HSV1-tk gene therapy protocols, identifying the location and magnitude of HSV1-TK enzyme activity by noninvasive imaging would provide a highly desirable measure of gene expression (following successful gene transduction). Importantly, the acquired sensitivity of HSV1-tk-transduced cells to GCV provides an additional margin of safety, because the transduced cells can be easily eliminated by treatment with GCV.

The wild-type HSV1-tk and HSV1-sr39tk the are the most widely used reporter genes for nuclear (PET) imaging, and the two most widely used radiolabeled reporter probes are $\left[{ }^{124} \mathrm{I}\right] \mathrm{FIAU}$ and $\left[{ }^{18} \mathrm{~F}\right] \mathrm{FHBG}$, respectively. The ability to image "late", 24 or more hours after [ [ ${ }^{124}$ I]FIAU administration, provides an additional advantage; namely, the opportunity to achieve high image specificity due to physiological washout of background radioactivity and the retention of the radioactivity in HSV1-tk transduced tissue. Also, the HSV1-tk/eGFP fusion reporter gene (TKGFP) was developed, which allows for the optical microscopic and whole body fluorescence imaging as well as PET imaging [39]. In recent reports by Doubrovin et al. (2001) [40] and Ponomarev et al. (2001) [41], the advantages of TKGFP reporter gene have been demonstrated in multi-modality imaging of p53-dependent transcription of endogenous genes and T-cell activation, respectively.

Jacobs, et al. (2001) for the first time reported a successful imaging of adenoviral mediated direct intratumoral HSV1-tk gene delivery and expression in human patients with glioblastoma with $\left[{ }^{124} \mathrm{I}\right] \mathrm{FIAU}$ and PET [42]; Yaghoubi, et al (2001) reported on human pharmacokinetics of $\left[{ }^{18} \mathrm{~F}\right] \mathrm{FHBG}$ [43-45] and PET imaging in healthy volunteers. Most recently, Peñuelas, et al. (2005), demonstrated successful imaging of adenoviral vector-mediated delivery and expression of HSV1-tk gene in patients with liver cancer (Figure A). Therefore, clinical PET imaging of HSV1-tk reporter gene expression for monitoring the fate of HSV1-tktransduced progenitor cells should be feasible. 


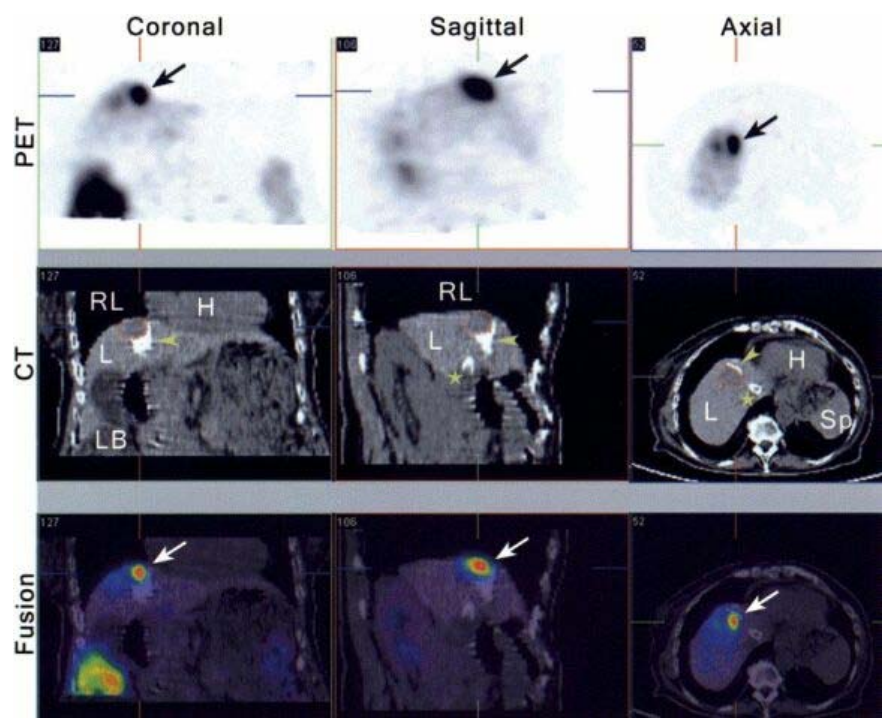

Figure A. PET-CT imaging of HSV1-tk transgene expression in humans. Columns 1 to 3 show the $5-\mathrm{mm}$ thick coronal, sagittal, and transaxial slices, respectively, from a [18F]FHBG-PET-CT study in patient 5. All sections are centered on the treated tumor lesion (dotted lines in the CT images) and show [18F]FHBG accumulation at the tumor site (arrows). Anatomicmetabolic correlation can be obtained by fused PET-CT imaging. The white spots on the liver seen on the CT images correspond to lipiodol (arrowheads) retention after transarterial embolization of the tumor and a trans jugular intrahepatic portosystemic shunt ( $\star$ ). Tracer signal can be seen in the treated lesion (arrows), whereas no specific accumulation of the tracer can be seen in the necrotic, lipiodol-retaining regions around it. $H$, heart; $L$, liver; $L B$, large bowel; $R L$, right lung; $S p$, spleen. 


\section{PROGRESS REPORT.}

Background. Tumor progression involves tumor cell proliferation and infiltration into surrounding tissue, and induction of different adaptive pathophysiologic and pathomorphologic processes in tissues that become involved in the developing tumor stroma. Tissue remodeling and neo-vascularization are critical processes in tumor stroma development (47). Recent studies have identified three main mechanisms of tumor angiogenesis: (a) existing vessel cooption (48); (b) endothelial sprouting (49); and (c) systemic recruitment of bone marrow-derived progenitor cells including endothelial and mesenhymal progenitor cells (50). Mesenchymal stem cells (MSC) derived from bone marrow (51) are capable of self-renewal and differentiation into various somatic lineages (52). The in vivo plasticity of MSC has been utilized in novel therapeutic approaches to repair structural damage of the bone (53), central nervous system (54), skin (55), blood vessels (56) and myocardium (57).

Previous studies have demonstrated that the adoptively transferred MSCs can migrate and engraft into the stroma of established tumor lesions (58). This phenomenon forms the basis for the paradigm of "Trojan Horse" approach, in which the adult stem cells are used as shuttle vectors for delivery of gene therapies into growing tumors. If proven clinically effective, such an approach may in the future provide the means for therapy of metastatic tumors. However, from the clinical perspective it is critical to demonstrate that such an approach is not limited to radiologically reliably detectable tumors, but can also be used to target minimal residual disease and micro-metastatic tumors. Therefore, in the current study the aim was to investigate whether the MSCs after systemic intravenous administration will migrate and engraft into the microscopic tumor lesions during early stages of their development. Another question explored was whether the proliferation of engrafted MSCs in the developing tumors can generate a significant fraction of tumor stroma, which could be subsequently eliminated with suicide gene therapy (e.g., HSV1-tk with ganciclovir, GCV), or provide the source for production of various anti-tumor cytokines such as INF $\beta$ (58) or IL2 (59).

Project Progress. To start addressing these questions, the immortalized human MSCs were used that were lentivirally transduced with the herpes virus type one thymidine kinse, HSVTK, and the A.victoria enhanced green fluorescent protein, eGFP, under control of two separate promoters. As in case of previously described TKGFP fusion reporter gene (60-63), the eGFP reporter gene facilitated the FACS-aided selection of transduced MSCs and their fluorescence microscopic visualization in vitro and in situ. The HSVTK reporter gene allowed for the repetitive molecular imaging with 18F-FHBG micro-positron emission tomography (microPET) for non-invasive in vivo whole body monitoring of the migration, engraftment, and proliferation of intravenously administered MSCs into tumor stroma or other organs and tissues.

Both the non-invasive repetitive microPET imaging and the in situ analyses of tumor tissue samples revealed that hMSC localized to the sites of microscopic tumor lesions and participated in tumor stroma formation. The latter process included initial proliferation of hMSCs and their subsequent differentiation into vascular endothelial and other tumor stromal cells, as well as into different cellular components of peritumoral tissues (i.e., skin and hair follicles).

Tumor Cells. The invasive variant f HT-29 Inv2 cells (64) was propagated in an in vitro Matrigel ${ }^{\mathrm{TM}}$ (BD Biosciences, MA, USA). The CCS line is a human colon adenocarcinoma. Both cell lines were grown in minimal essential medium (MEM) containing $10 \mathrm{U} / \mathrm{ml}$ penicillin, $10 \mu \mathrm{g} / \mathrm{ml}$ streptomycin, $2 \mathrm{mM}$ glutamine and $10 \%$ fetal bovine serum (FBS, Hyclone), in 37oC humidified atmosphere with $5 \% \mathrm{CO} 2$.

Human mesenchymal stem cells. The hMSC line was originally cultured from the bone marrow of a 61year old female donor and immortalized by retroviral-mediated transduction of human papilloma virus E6/E7 genes, and maintained in Dulbecco's modified Eagle's medium containing $1 \mathrm{mg} / \mathrm{ml}$ glucose and $10 \%$ FBS of a selected lot (Gibco) (65). This immortalized hMSC line is more than 99\% positive for CD29, CD44, CD90, CD105, SH2 and SH3 MSC-characteristic markers.

Lentiviral vector construction and transduction. The self-inactivating (SIN) pTY-EFeGFP lentiviral vector backbone was obtained from Dr. Lung-Ji Chang, Univ. of Florida (65). The pTY-EFeGFP-TK vector, was constructed by inserting into the herpes simplex virus type-1 thymidine kinase gene (HSVTK) driven by the HSV1-tk promoter into the Kpnl site of the pTY-EFeGFP vector that carries the eGFP reporter gene driven by the EF1á promoter. Using the TE671 vector at MOI of one, the eGFP expression was observed in close to $30 \%$ of hMSC. The transduced eGFP+ (and HSVTK+) hMSCs were separated using FACS Vantage (BD BioScience, CA) and designated TG-hMSC "tracer cells". Subsequently, the expression of HSVTK in TGhMSCs was verified using in vitro radiotracer accumulation assay with [18F]FHBG $(62,67)$, and by an in vitro susceptibility test to GCV (LD50 of $0.01 \mathrm{mM}$ ) as previously described $(23,24)$. In contrast, non-transduced 
hMSCs and non-transduced human colon cancer cells were insensitive to GCV at $1 \mathrm{mM}$ and showed negligible uptake and retention of [18F]FHBG in vitro.

Tumor xenografts in mice. The mice were used for experiments at 6-10 weeks of age. HT-29 Inv2 or CSS tumor cells were injected subcutaneously (s.c.) at a dose of $10^{6}$ cells per injection site with or without tracer TG-hMSCs, as will be described further. Tumor nodules usually became palpable within 1 week and reached about 4-6 $\mathrm{mm}$ in diameter in 4 weeks after s.c. cell injection.

Micro-PET imaging. The [18F]FHBG was prepared using no-carrier added one-pot synthesis procedure, as previously described (70). Tumor-bearing mice under gas anesthesia ( $2 \%$ Isoflurane / $98 \%$ oxygen) were injected via tail vein with $230-250 \mu \mathrm{Ci}$ of [18F]FHBG in $0.2 \mathrm{ml}$ sterile saline and imaged on a micro-PET R4 system (Concorde Microsystems Inc., Knoxville, TN) at 4 hours after radiotracer injection. Axial PET images were acquired for 10 minutes in prone position of mice. The images were reconstructed by using $2 \mathrm{D}$ ordered subsets expectation maximization (2D-OSEM) as previously described (71).

Histology and immunohistochemistry. In different study groups, selected mice were sacrificed after the end of PET imaging session by cervical dislocation. Subcutaneous tumors were dissected from the surrounding soft tissues and fixed in $2 \%$ paraformaldehyde for fluorescent microscopy of eGFP (72). Alternatively, tissue sections were immunostained for eGFP expression using the rabbit anti-GFP polyclonal antibody (Clontech, CA), followed by horseradish-labeled goat anti-rabbit IgG antibody and finally processed for immunoperoxidase-DAB staining (Vector, CA) to facilitate the visualization of lower levels of eGFP expression.

Polymerase chain reaction. The nuclear DNA extracts from cultured cells and tumor tissue samples were prepared using commercially available DNA isolation kit (Blood \& Tissue Genomic Mini; Viogene). Polymerase chain reaction was performed with eGFP primer pairs (sense: 5'ATGAGCAAGGGCGAGGAACT-3'; antisense: 5'-TCACTTGTACAGCTCGTCCA-3') by initial heating at $950 \mathrm{C}$ for $5 \mathrm{~min}$, followed by 35 cycles of $950 \mathrm{C} 1 \mathrm{~min}, 60^{\circ} \mathrm{C} 1 \mathrm{~min}$ and $72^{\circ} \mathrm{C} 1 \mathrm{~min}$, and a final elongation reaction at $72^{\circ} \mathrm{C} 10 \mathrm{~min}$. DNA products were separated by electrophoresis in $1 \%$ agarose gel, stained with ethidium bromide, and visualized under UV illumination.

Cytofluorometry. Single cell suspensions were obtained from the aseptically minced tumor fragments by collagenase digestion in 15 volumes per wet weight of Hank's balanced solution containing $1 \mathrm{mg} / \mathrm{ml}$ collagenase and $2 \mathrm{mM}$ EDTA overnight at $4 \mathrm{oC}$. The fractionated cells were washed with PBS and used directly or cryopreserved in FCS:DMSO $(9: 1 \mathrm{v} / \mathrm{v})$ at $-80^{\circ} \mathrm{C}$ before analysis. The washed cells, with or without fixation/permeabilization, were reacted with rabbit anti-GFP polyclonal antibody (Clontech) plus FITC-labeled donkey anti-rabbit IgG antibody, or FITC-labeled anti-GFP; mouse anti-human von Willebrand factor (hvWF; Immunotech) plus PE-labeled goat anti-mouse IgG antibody or PE-labeled anti-hvWF; PE-labeled mouse monoclonal antibodies against human CD14 (Pharmingen), CD31, CD90, CD105 (Serotec) and smooth muscle actin (Chemicon). Isotype control antibodies were utilized in parallel to set negative gates. The cells were analyzed on a FACS Vantage cytofluorometer using Cell Quest software (BD Biotech).

Micro-PET imaging of human mesenchymal stem cell targeted tumors. In the first group of mice $(\mathrm{N}=6)$, the ability of MSCs to proliferate after co-implantation with tumor cells inside the growing tumor lesions and contribute to the development of tumor stroma was studied. In this group, two s.c. tumor xenografts were established by s.c. injection of a mixture of $1 \times 10^{6} \mathrm{HT}-29$ Inv2 cells and $5 \times 10^{5}$ tracer TG-hMSCs [HT-29+TGhMSC] into the right flank, and by s.c. injection of $1 \times 10^{6}$ HT-29 Inv2 cells alone [HT-29] into the left flank. The micro-PET imaging with [18F]FHBG was performed 4 weeks later and demonstrated a profound uptake and retention of the radiotracer in HT-29+TG-hMSC tumors $(5.78 \pm 4.79 \% \mathrm{ID} / \mathrm{g})$, indicating persistent HSVTK expression by the tracer TG-hMSC-derived tumor stroma (Fig. 1A). In contrast, no [18F]FHBG uptake and retention above body background was observed in the contralateral HT-29 tumors, which served as negative control. 
In the second group of mice $(\mathrm{N}=6)$, only one tumor xenograft per mouse was established by s.c. injection of $1 \times 10^{6}$ HT-29 Inv2 cells into the right flank. In this group, $5 \times 10^{5}$ tracer TG-hMSCs were administered through the tail vein three days after s.c. tumor injection. In this group, micro-PET imaging studies confirmed the ability of intravenously administered tracer TG-hMSCs to migrate to the sites of microscopic tumor lesions, engraft into these microscopic tumor lesions, and contribute to the development of a significantly portion of tumor stroma (Fig. 1B).

Similar results were observed in tumors produced from human colon adenocarcinoma cell line, CCS. Micro PET images obtained at different days after s.c. injection of tumor cells demonstrated the initial migration of intravenously administered tracer TGhMSC to the sites of s.c. CCS tumor cell injection. Repetitive microPET imaging performed at 15 and 27 days after tracer TG-hMSCs injection (18 and 30 days of tumor growth) demonstrated exponentially increasing $18 \mathrm{~F}-\mathrm{FHBG}$ accumulation at the sites of s.c. CCS tumor cell injection (Fig. 1C, D), which is indicative of tracer TG-hMSC proliferation and significant contribution to growing tumor stroma development.

In the third group of mice $(\mathrm{N}=6)$, studies were aimed to determine whether the tracer hMSC-derived tumor stromal cells retain stem cell properties (i.e., the capacity for self-renewal). To address this question, tumor tissue samples were obtained at the end of a 30-day imaging study conducted in the second group of animals, and implanted s.c. aseptically minced tumor fragments of about $2 \mathrm{~mm}$ in diameter into the new NOD-SCID mouse recipients. Four weeks later, the recipient mice developed s.c. tumors and were imaged with [18F]FHBG PET, which demonstrated the lack of radiotracer uptake above the body background levels (Fig. 1E), which is indicative of a very low density or a lack of tracer TG-hMSCs progeny inside newly developed tumor stroma.

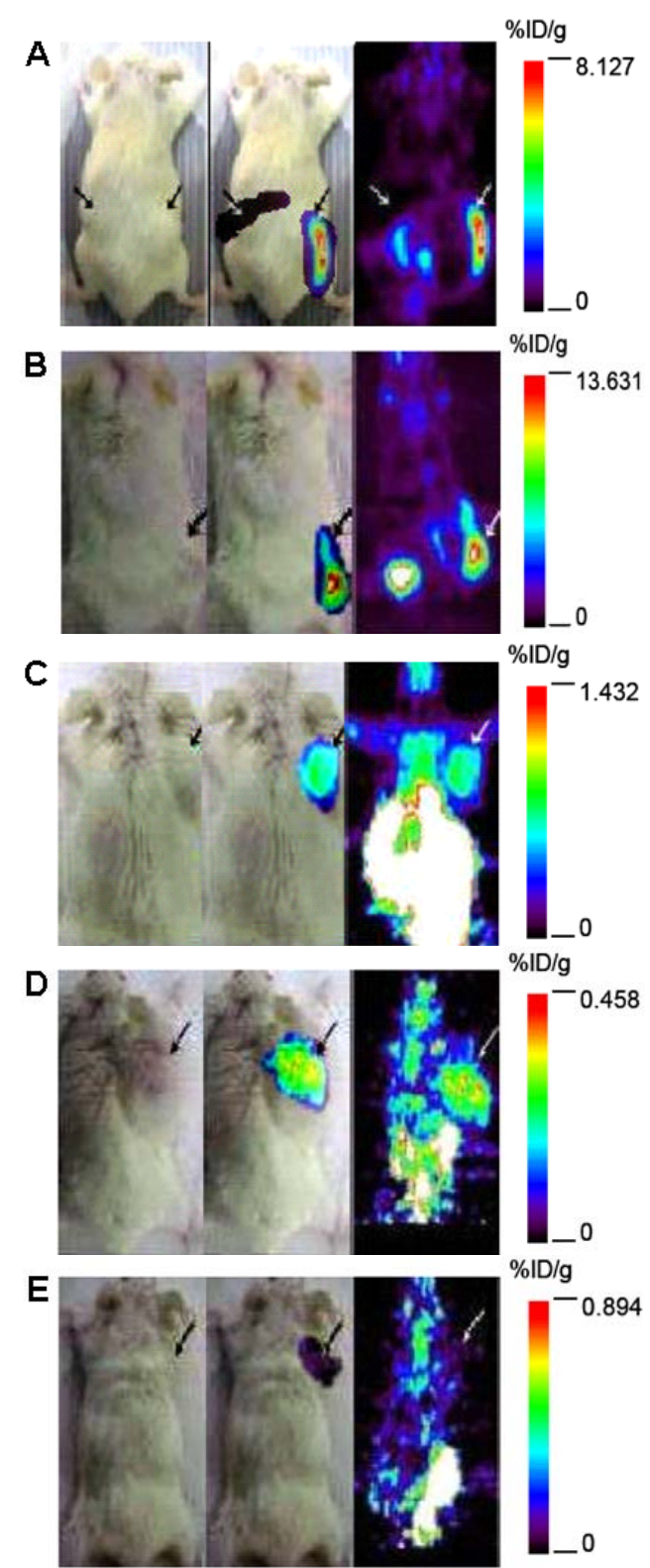

Figure 1. Micro-PET of colon carcinoma xenografts with or without s.c. co-injection of TG-hMSCs in NOD-SCID mice. [A] A mouse 30 days after inoculation of $1 \times 10^{6}$ HT-29 Inv2 cancer cells plus $5 \times 10^{5}$ TG-hMSCs (HT29 (s.c.)+TG-hMSC(s.c.) tumors) in the right flank and $1 \times 10^{6}$ HT-29 Inv2 alone (HT-29(s.c.) tumors) in the left flank. Micro-PET images showed [18F]FHBG in HT29(s.c.)+TG-hMSC(s.c.) tumors but not in contralateral HT-29(s.c.) tumors (arrows). [B] A mouse 30 days after s.c. injection of HT-29 and 27 days after i.v. infusion of TG-hMSC (HT-29(s.c.)+TG-hMSC(i.v.) tumors). MicroPET image of HT-29(s.c.)+TG-hMSC(i.v.) tumors at 3 hours after [18F]FHBG infusion. [C \& D] Micro-PET images of mice, injected s.c. with $2 \times 10^{6}$ CCS cells and 3 days later injected i.v. with $5 \times 105$ tg-hMSC. Micro-PET image showing an increase in [18F]FHBG accumulation in tumors between 18 days [C] and 30 days [D] of tumor growth. [E] Micro-PET image showing no [18F]FHBG retention in a tumor formed in a new NOD-SCID mouse host at 4 weeks after transplantation of minced CCS(s.c)+TG-hMSC(i.v.) tumor fragments obtained from the mouse shown in panel $D$. 
Microscopic analyses of tumor stroma and surrounding tissues. To validate the results of non-invasive in vivo microPET imaging and to further characterize the type of cellular elements in tumor stroma, tumor tissue sections obtained in all groups of animals were examined for the type of cells expressing eGFP. In the first group of mice with s.c. coinjection of tumor cells and tracer TG-hMSCs, as well as in the second group with i.v. administration of tracer TG-hMSCs, the eGFP fluorescence and immunostaining were detectable mainly in cells forming the capillarylike structures (Fig. 1 A,B, G,H,I), in the cells within the inner layer of small arterioles (Fig. 2 B, C), and in connective tissue cells (Fig.2 F). These results demostrate that both intratumorally co-administered as well as intravenously administered tracer TG-hMSCs contributed significantly to the development of vascular and connective tissue components of tumor stroma growing in NOD-SCID mice. Surprisingly, a relatively large number of tracer TGhMSCs were detected the skin

adjacent to and overlaying the s.c. tumors. In particular, eGFPexpressing cells were identified in hair follicles, the basal layer of dermis, and in the endothelial and intimal layers of small blood vessels in the skin overlaying s.c. tumors (Fig. 3).

In situ validation of PET imaging of tracer hMSC proliferation in tumors. To validate microPET imaging results and to quantitate the magnitude of tracer TG-hMSCs proliferation in tumors in different study groups, fresh tumor tissue samples were gradually digested to obtain single cell suspensions containing both the tumor and stromal cell fractions. The numbers of eGFP+ cells were assessed by cytofluorometry and plotted over time (days of tumor growth in vivo) (Fig. 4). Because only $5 \times 10^{5}$ tracer hMSCs were initially administered either by co-implantation or by i.v. injection, these data indicate that tracer TG-hMSCs had proliferated at least several times during the tumor stroma development. This was more evident when the cell numbers were fitted with an exponential function. The rates of tracer TG-hMSC-derived

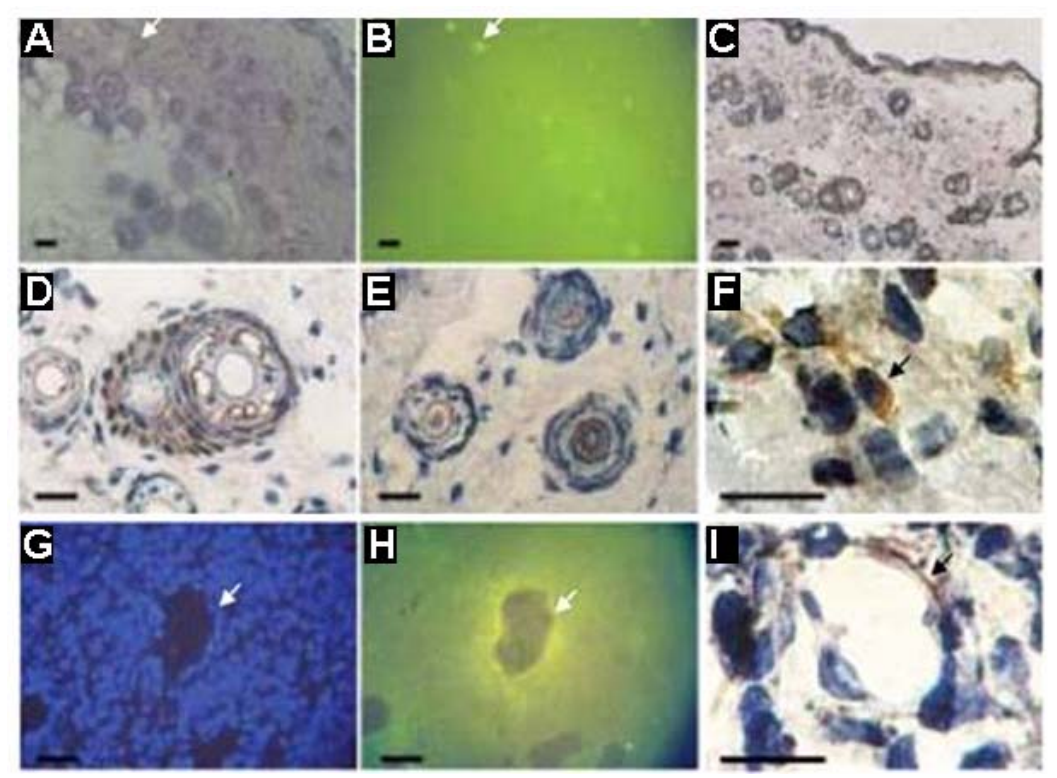

Figure 2X. Histological characterization of eGFP-expressing cells in tumors developed from s.c. injection of HT-29 Inv2 cells and tracer TGhMSC in NOD SCID mice (HT-29(s.c.)+TG-hMSC(s.c.) tumors). Light microscopy (x20 mag.) of the H\&E stained sections of HT-29(s.c.)+TGhMSC(s.c.) tumors at 30-days of growth (A). The distribution of eGFP+ fluorescent cells in adjacent sections examined by fluorescence microscopy $(B, H)$ and immunoperoxidase $(C, D, E, F, I)$. Numerous eGFPpositive cells were detected in the periphery of tumors (A-C) and in hair follicles (D, E). Toward the inner tumor, the GFP-positive cells were fewer and occasionally observed in the tumor stroma $(F)$ but were often restricted to small vessels ( $G$ - DAPI stain of area $H$ ) and dispersed within the parenchyma. At higher magnification, the GFP-positive cells appeared as small or elongated mononuclear cells, with vascular endothelial cell-like morphology, lining the inner walls of small vessels and forming capillaries (I). Scale bars $=20 \mu \mathrm{m}$.

Figure 3. The morphology of 30-day HT-29(s.c.)+TG-hMSC(i.v.) tumors. Numerous eGFP-containing cells revealed by immunoperoxidase staining were located mainly within dermis and hair follicles of the skin overlaying the s.c. tumors $(A)$ and also diffusely present in the inner wall of small arteries but not in the smooth muscle layers (B). At higher magnification, the cells had small and ellipsoid nuclei, characteristic of the vascular endothelial cells (C). Adjacent tissue sections stained with the omission of the anti-GFP antibody were used as negative controls (D-F). Scale bar = $20 \mu \mathrm{m}$.

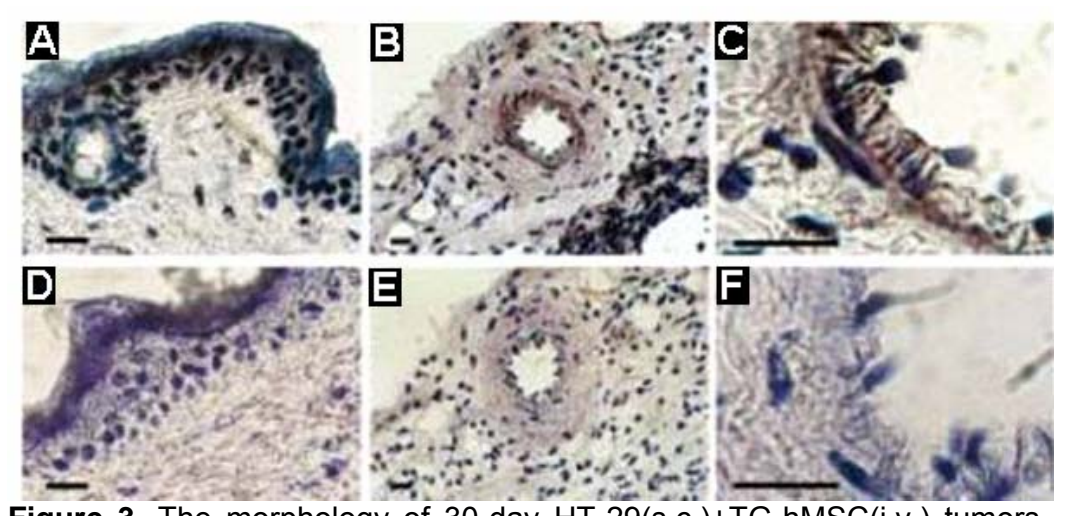


progeny cell proliferation were similar in different study groups independent of the mode of administration. The average doubling time of tracer TG-hMSCs was $6.51 \pm 0.36$ days, as determined by dividing the $\operatorname{Ln}(2)$ by the coefficients of exponential fits of data. The levels of microPET detectable 18F-FHBG radioactivity concentration in CCS(s.c.)+TGhMSCs(i.v.) tumors increased exponentially between days 17 and 30 and paralleled increasing numbers of eGFP+ cells in the growing tumors.

The sensitivity of microPET imaging of tracer TG-hMSCs could be calculated by dividing the number of recovered GFP+ cells from s.c. tumors by the corresponding tumor volume. For example dividing $3.2 \times 10^{6}$ TGhMSCs recovered from the day-18 CCS(s.c.)+TG-hMSCs(i.v.) tumors by their volume of about $120 \mathrm{~mm}^{3}$, results in $2.6 \times 10^{4}$ cells $/ \mathrm{mm}^{3}$, that were reliably detectable by the microPET R4 after injection of $250 \mu \mathrm{Ci}$ 18FFHBG per animal. Because the microPET R4 can detect at least a 10-20 fold lower levels of radioactivity concentration, it can reliably extrapolated that the lower threshold of microPET imaging is close to 1000 cells $/ \mathrm{mm}^{3}$.

In situ quantitation of TG-hMSC progeny cells and cell surface markers. In situ characterization of eGFP+ cells in HT-29(s.c.)+TG-hMSC(i.v.) tumors involved PCR amplification of eGFP sequence (717 bp) using $1 \mu \mathrm{g}$ of cellular DNA prepared from digestion-aided disaggregates of $\mathrm{HT}$ 29(s.c.)+TG-hMSC(i.v.) tumors and HT-29(s.c.) tumors. In HT-29(s.c.)+TG-hMSC(i.v.) tumors, the eGFP gene was barely detectable in readily dissociated, loose tumor cell fractions, but was abundant in the stromal cell fraction. In contrast, eGFP gene was not detectable in tumors established from HT-29 cells only, without co-administration of TG-hMSCs. The TG-hMSC and HT-29 tumor cells were used as positive and negative controls, respectively (Fig. 5A).

Cytofluorometric analysis of HT-29(s.c.)+TG-hMSC(i.v.) tumors demonstrated that $11.5 \%$ of all cells were eGFP+ (Fig. 5B). Antibodies against various human cell surface markers were used to analyze the phenotype of the eGFP(+) cells by immunocytofluorometry. An endothelial cell marker, von Willebrandt factor, was found on about one third of the eGFP(+) cells of HT29(s.c.)+hMSC(i.v.) tumors (Fig. 5C). Elevated levels of CD31 endothelial marker (73) expression, but low and undetectable levels of CD90 and CD105 were observed in stromal matrix-associated eGFP(+) cells (Fig. 5C), although the CD90 and CD105 are known to be highly expressed in hMSCs (74). None of the eGFP(+) cells reacted with anti-human smooth muscle actin antibodies (data not shown). Thus, the targeted tg-hMSC had differentiated mainly into the endothelial but not the smooth muscle cell components in tumor angiogenesis, confirming prior immunohistochemical observations (Figs. 2 and 3).

Apparent loss of self-renewal capacity of differentiated tracer TG-hMSC-derived stromal cells. To determine if tumor-engrafted tracer hMSC-derived progeny stromal cells retain self-renewal capacity, tumor and stromal cells obtained from digested tissue fragments of micro-PET positive CSS(s.c.)+hMSC(i.v.) tumors were cultured in vitro. After five days in culture, the eGFP+ fluorescent cells were visible within spheroid-like cellular clusters and flat colonies (Fig. 6A-D). The eGFP expression in these cultured cells was confirmed by immunocytofluorometry (Fig. 6E,F). However, these eGFP-expressing cells did not proliferate further under these culture conditions and were rapidly overgrown by the CCS colon cancer cells. These 
results are consistent with our observations with microPET imaging in experiments involving re-implantation of small tumor fragments into new recipient mice (the third study group; Fig. 1E).
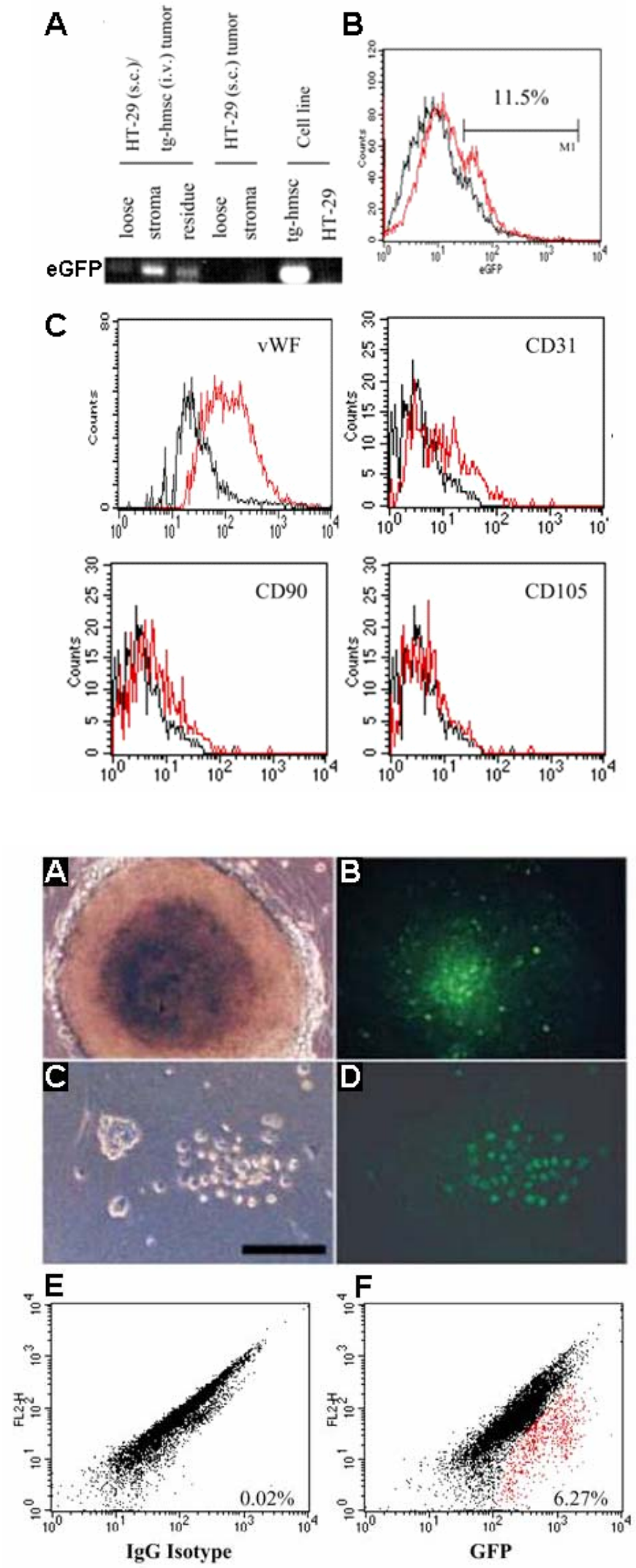

Figure 5. In situ characterization of eGFP+ cells in HT29(s.c.)+TG-hMSC(i.v.) tumors. [A] Agarose gel electrophoresis of PCR-amplified eGFP sequence (717 bp) performed using $1 \mu \mathrm{g}$ of cellular DNA prepared from loosely associated cell fraction (loose), stromal matrix associated fraction (stroma) and the residue (residue) of HT-29(s.c.)/tg-hMSC(i.v.) tumor and HT-29(s.c.) tumor, tg-hMSC and HT-29 cells. [B] Cytofluorometric histograms comparing the stromal matrix cell fractions of HT-29(s.c.)+TG-hMSC(i.v.) tumors (red) and HT-29(s.c.) tumors (black), examined with anti-GFP specific antibody. eGFP+ cells in the gated area represented $11.5 \%$ of total cells. [C] Histograms showing the presence of human endothelial cell markers but absence of hMSC markers in eGFP(+) cells obtained from the HT29(s.c.)+TG-hMSC(i.v.) tumor after 30 days of growth in vivo. Stromal matrix-associated cell fractions were double-stained with FITC-labeled anti-GFP mouse monoclonal antibody, and with a PE-labeled anti-vWF, or anti-CD31, or anti-CD90, or anti-CD105 to assess the expression of these cell surface markers on eGFP+ cells (red histograms). PE-labeled mouse isotype immunoglobulins were included in parallel to serve as the negative control (black histograms).

Figure 6. In vitro assessment of self-renewal capacity of differentiated tracer TG-hMSC-derived stromal cells. The morphology of digestion-aided disaggregated tissue fragments of micro-PET positive CSS(s.c.)+hMSC(i.v.) tumors after five days in culture as seen by dark field microscopy (A, C) and fluorescence microscopy of eGFP $(B, D)$. The eGFP+ fluorescent cells are clearly visible within spheroid-like cellular clusters $(A, B)$ and flat colonies (C, D). The eGFP expression in these cultured cells was confirmed by immunocytofluorometry using antieGFP antibody (F); the lgG isotype antibody was used for control $(E)$. 
Studies described above were conducted to assess whether the hMSCs can migrate and engraft into the microscopic tumors, subsequently proliferate and form a significant portion of developed tumor stroma. A clinically applicable in vivo whole body PET imaging with 18F-FHBG was used to non-invasively monitor the fate of genetically modified HSVTK and eGFP expressing "tracer" hMSCs (TG-hMSCs) upon their intratumoral or intravenous administration. The term "tracer cells" is being used in context of the potential future clinical scenario, in which the HSVTK-expressing hMSCs will be used to "trace" the co-administered therapeutic hMSCs genetically modified to express various anti-cancer cytokines $(58,59)$ Similar PET imaging approach was applied in several recent studies for non-invasively visualization of HSVTK expressing stem cells after direct intra-myocardial injection $(75,76)$, and for visualization of tumor targeting by the adoptively transferred tumor antigen-specific T cells $(77,78)$. Another potential clinical therapeutic protocol, which would be greatly facilitated by non-invasive imaging, would include repetitive (e.g., bi-weekly or monthly) administration of HSV1-TK expressing autologous stem cells to patients following resection of a primary tumor which has high likelihood of metastases that are radiologically not detectable at the time of resection. In case the local or distal metastases will be developing after resection of primary tumor, such repetitive administration of HSV1-TK expressing stem cells would result in development of HSV1-TKexpressing tumor stroma, which should be detectable with whole body PET imaging. The HSV1-TK expressing tumor stroma could potewntially be "eliminated" by treatment with GCV, which should cause a significant regression of metastatic tumor lesions, the efficacy of which can be assessed by conventional anatomic CT, MR, and [18F]-FDG imaging. Thereafter, the cycle of therapy with HSV1-tk expressing stem cells followed by whole body PET imaging of HSV1-tk expression and GCV therapy could be repeated.

As the initial step of our studies, the sensitivity and specificity of micro-PET imaging with [18F]-FHBG was assessed for the detection of HSVTK expressing tumor stroma in subcutaneous tumors that had been established by s.c. co-injection of colon HT29 carcinoma cells and tracer hMSCs in mice. The sensitivity of microPET imaging with [18F]-FHBG depends on the level of HSVTK reporter gene expression per cell and the number of HSVTK+ cells within the minimum resolution volume of $2 \times 2 \times 2 \mathrm{~mm}$ for the MicroPET R4 scanner (Concorde, TN). The calculated lower threshold of microPET R4 sensitivity in our in vivo imaging in situ validation studies was close to 1000 cells $/ \mathrm{mm}^{3}$, which is comparable to results reported recently by Gambhir's group (Stanford Univ., CA), which estimated the lower threshold of MicroPET R4 scanner sensitivity for imaging with [18F]-FHBG sr39HSVTK-expressing tracer cells as $10^{4}-10^{5} / \mathrm{mm}^{3}$ of tissue (76).

Next, in vivo PET imaging and in situ analyses were performed at 15 and 27 days after i.v. administration of HSVTK+, GFP+ tracer hMSCs in mice with microscopic s.c. CCS tumors. We demonstrated that these cells engrafted into the microscopic tumors, subsequently proliferated with a calculated average doubling time of $6.51 \pm 0.36$ days, and contributed to the formation of a significant fraction of tumor stroma. Tumortargeting properties of stem cells have been previously reported in glioblastoma model (79); in studies of tumor angiogenesis by hematopoietic stem cells (50); stem cells were used as vehicles for gene therapy of human colon cancer xenografts (58). Cancer cells are known to produce various growth factors which stimulate angiogenesis (80). In response to these factors, endothelial precursors including bone marrowderived endothelial progenitors (56), and hematopoietic stem cells (50) may become recruited into tumor neo-vascularization process. Whether some of these factors possess chemotactic and/or selective differentiation-inducing activities for MSC remains to be investigated. It remains to be determined whether the endogenous MSCs are mobilized by the microscopic tumors from the bone marrow and migrate to sites of microscopic tumors, and what percentage of tumor stroma develops from the engrafted MSCs?

Our in vivo PET imaging observations were cross-validated by in situ and in vitro analyses. Specifically, the immunocytofluorometric analyses of tumors confirmed the presence of eGFP+ progeny cells differentiated into endothelial cells of tumor capillaries and small blood vessels, hair follicle cells and basal cells of dermis overlaying the s.c. tumor xenografts. The later observations may be explained, at least in part, by the capacity of stem cells to non-specifically home into the sites of tissue damage and inflammation (81), which are often observed in the skin overlaying s.c. tumors.

Previously, it was demonstrated that MSCs have propensity to differentiate as osteogenic, chondrogenic, adipogenic and neurogenic lineages in vitro $(74,82)$. It is not known if trans-differentiation from hMSC to epithelial cells at the tumor site could involve mechanisms such as the recently reported in vitro fusion of hMSC with heat-shocked epithelial cells (55). These differentiated tracer hMSC-derived progeny cells had exhibited a limited capacity for self-renewal as evidenced from re-transplantation experiments. One possible explanation of these results is that HSVTK+, GFP+ tracer stem cells which contributed to the development of 
the tumor stroma in the original host, subsequently diminished their proliferative capacity due to differentiation, which was also evident from our in vitro re-plating experiments. Another possible explanation is that by four weeks post transplantation into the new host the HSVTK+, GFP+ tracer stem cells from the transplanted tumor tissue became "diluted" within the newly developing tumor stroma and/or were substituted by the recipient host-derived stroma-forming cells. Similar results on limited renewal capacity and less than two weeks life-span of tumor-derived endothelial cells in re-transplanted tumors have been reported previously (83).

In conclusion, the current study provides direct evidence for microscopic tumor targeting by exogenously administered hMSCs. The intravenously administered HSVTK-expressing tracer hMSCs can be used for non-invasive monitoring of stem cell-based therapies using whole body PET imaging with 18F-FHBG or other HSVTK-specific radiotracers $18 \mathrm{~F}-\mathrm{FEAU}$ and 18F-FFAU $(84,85)$ or $124 \mathrm{I}$-FAIU $(86,87)$. It is also conceivable that PET imaging of intravenously administered HSVTK+ tracer hMSCs can be exploited as a novel approach for noninvasive detection of otherwise radiologically non-detectable microscopic tumors and for monitoring the formation of HSV1-tk expressing tumor stroma for GCV therapy. Studies outlined in the current proposal aim to validate this pro-drug activation stem cell therapeutic approach in preclinical setting as a prelude to clinical translational studies, which are planed to be conducted at the MD Anderson Cancer Center in the future.

Non-invasive imaging of cellular trafficking during bone marrow transplantation. In another recent study, T-cells had been retrovirally transduced with HSV1-tk reporter gene and co-transplanted them $\left(4 \times 10^{6} \mathrm{~T}\right.$ cells/animal) with $4 \times 10^{6}$ T-cells/animal bone marrow progenitor cells to irradiated allogenic recipient mice. These mice developed different degrees of GVHD at 13 to 24 days post-transplant, which was manifested by different degrees of T-cell infiltration in the spleen and gut of these animals, as visualized on microPET images obtained with i.v. ${ }^{18} \mathrm{~F}-\mathrm{FEAU}\left(150 \mathrm{\mu Ci} /\right.$ animal). Cross-sectional axial, coronal, and saggital ${ }^{18} \mathrm{~F}-\mathrm{FEAU}$ PET images are presented in the Figure 7 and demonstrate highly specific localization of HSV1-tk expressing cells in the spleen (animal A) and gut (animal B), consistent with a classical GVHD pattern. The degree of infiltration and T-cell proliferation was consistent with the magnitude and extent of PET signal distribution as well as degree of animal morbidity.

These results and our previous studies on imaging T-cell as well as progenitor cell trafficking with PET $[40,41]$ indicate that clinical translation of such imaging for monitoring adoptive cells therapeutic approach should be feasible.
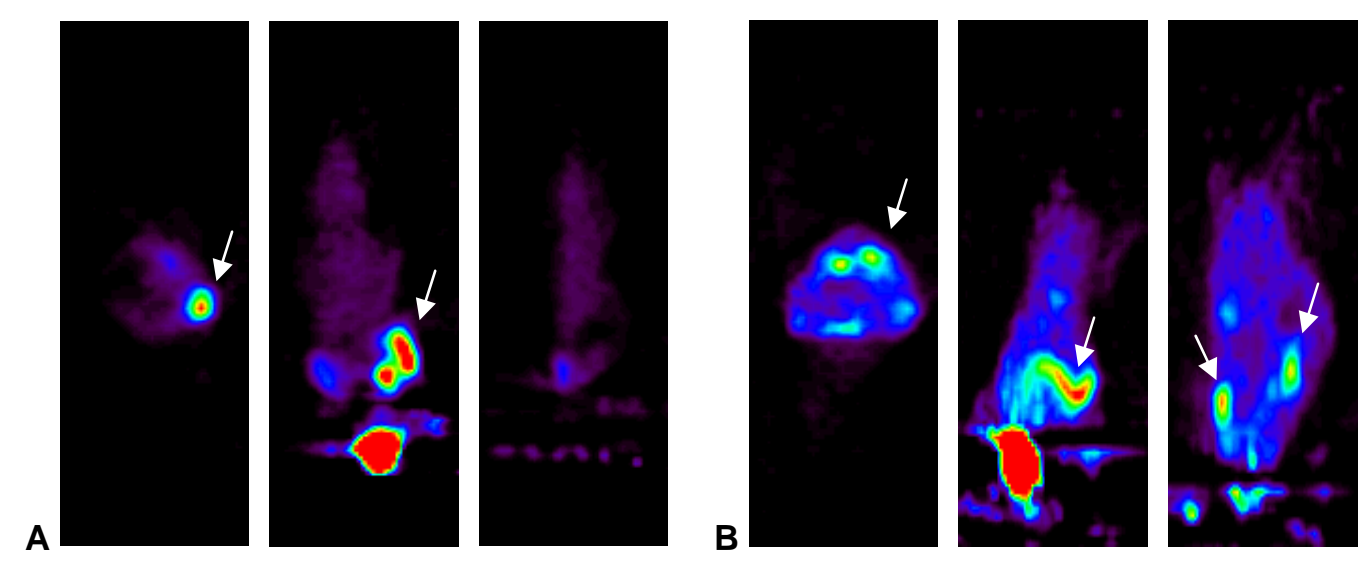

Figure 7. MicroPET imaging of HSV1-tk expressing allogeneic T-cells co-transplanted with the bone marrow progenitor cells for hematopoietic reconstitution. PET images from two different animals ( $A$ and $B$ ) demonstrate at 24 days post transplant the localization of HSV1-tk expressing cells in spleen (A) and gut (B) consistent with GVHD. 
Imaging Progenitor Cell Trafficking and Engraftment with BLI and PET/CT. Recently, we demonstrated that non-invasive multi-modality BLI and PET/CT imaging of TKGFP expression with $\left.{ }^{18} \mathrm{~F}\right] \mathrm{FEAU}$ in vivo is feasible for monitoring the dynamics of progenitor cell engraftment in living mice over several weeks of observation. In this study, CD34+ lineage negative progenitor cells were isolated from the C57BL/6 murine bone marrow and then transduced in vitro with a MoMLV-based retroviral vector to express the HSV1-tk/GFP (TKGFP) reporter gene for microPET/CT imaging as well as Luciferase gene for bioluminescence imaging (BLI). Subsequently, lin-, c-kit+, Sca-1+ and TKGFP+ cells were isolated using FACS, and 2.5x10 transduced "tracer" stem cells were administered intravenously into irradiated recipient C57BL/6 mice. Progenitor cell trafficking patterns and engraftment was initially monitored by BLI using D-luciferin, starting from the first hour post administration of "reporter" progenitor cells and performed every 2-3 days thereafter (Fig. 1). Also, PET/CT images with $\left[{ }^{18} \mathrm{~F}\right] \mathrm{FEAU}(100 \mu \mathrm{Ci} / \mathrm{animal})$ were acquired in parallel with $\mathrm{BLI}$ after the first 24 hours after administration of reporter-transduced progenitor cells and repeated every 2-3 days thereafter for two weeks. Preliminary data obtained over the period of monitoring by such repetitive noninvasive BLI and PET/CT imaging does reflect incredibly well the spatial and temporal dynamics of progenitor cell trafficking. Using both imaging modalities we have observed the temporal-anatomical checkpoints in such organs as the lungs (first hours) and spleen (7-9 days of rapid proliferation) and ultimate bone marrow targeting and engraftment. Shifting foci of engraftment were observed similar to those reported recently by Dr. Weissman's group at Stanford University (Stanford, CA) [1].
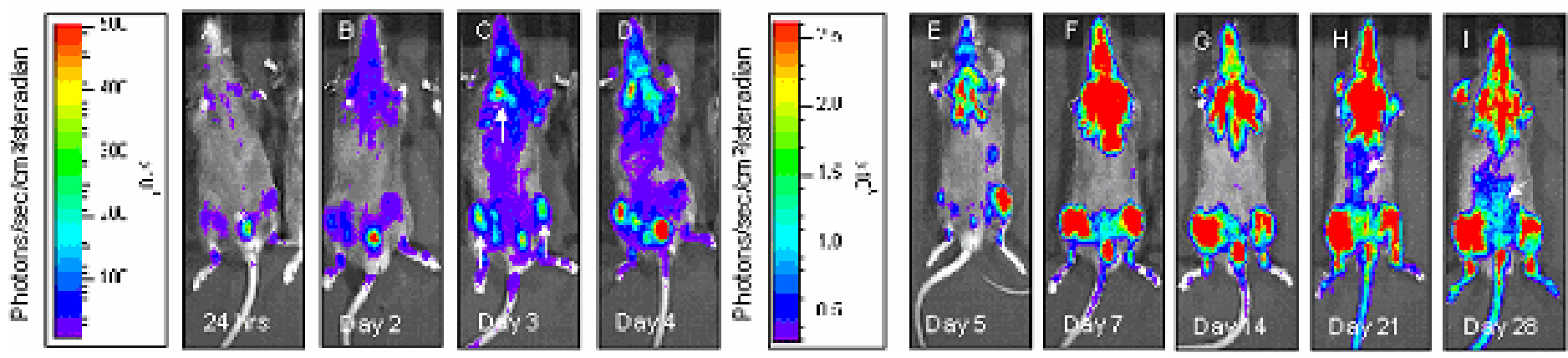

Figure 1. Bioluminescence imaging of Luc expressing (TKGFP+) progenitor cells in vivo in the same recipient mouse over time. The white arrows in the images obtained on days 21 and 28 after i.v. administration of progenitor cells indicate the sites of engraftment into the vertebrae. Despite the very high level of sensitivity of BLI, the "erosion" of anatomical resolution is evident from these serial BLI images, due to the light scattering properties of biological tissues. 


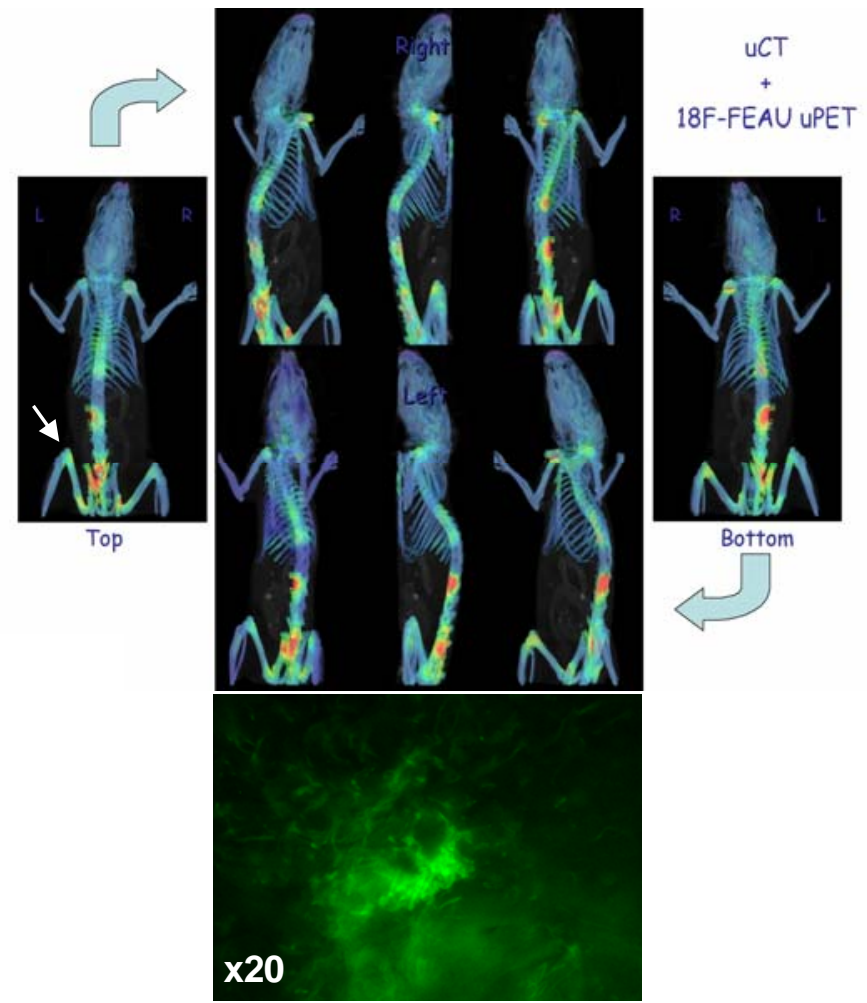

Figure 2. Maximum intensity projections of fused $\left[{ }^{18} \mathrm{~F}\right] \mathrm{FEAU}$ microPET/CT images of the same animal (as above) rendered in $3 \mathrm{D}$ and presented from different angles of view (day 6 post injection of $2.5 \times 10^{5}$ CD34+, lin-, c-kit+, Sca-1+, TKGFP+ progenitor cells and demonstrate the engraftment sites in the skeleton. Spleen signal was digitally removed to simplify the visualization of bone marrow-specific engraftment sites in 3D. The focus of bone marrow engraftment (arrow) of TKGFP positive cells is confirmed by fluorescence microscopy.

The early engraftment patterns were heterogeneous and variable in different animals during the first week of repetitive imaging. Uniformly, in all animals the stem cells trafficked into spleen, where the most of proliferative signal was observed during the 7-9 days post i.v. administration, followed by gradual increase of PET-detected signal from transduced cells engrafted into the bone marrow of long bones of limbs, sacrum, petroso-mastoid bones in the scull, and in the sternum (Fig. 2). More variable and patchy engraftment was observed in the vertebral column, which was evident during the two weeks of observation. Some animals were sacrificed after PET/CT imaging sessions on various days after progenitor cell administration to validate the results of noninvasive PET imaging by in situ analysis. The in situ fluorescence microscopic analysis confirmed the presence of TKGFP expressing bone marrow in the sites detected by PET imaging with $\left[{ }^{18} \mathrm{~F}\right] \mathrm{FEAU}$ (Fig. 2). The sensitivity of non-invasive PET imaging for quantitation of TKGFP-transduced cells with $\left[{ }^{18} \mathrm{~F}\right] \mathrm{FEAU}$ in vivo was very high, as demonstrated by the ability to reliably detect early fossae of engraftment consisting only of about ten thousand cells. Similar threshold of sensitivity of PET for quantitation (enumeration) of TKGFP transduced cells in vivo, $10^{6}$ per $100 \mu$ l of tissue (equivalent to $10^{4}$ cells/ $/$ l), was recently observed in $\mu$ PET imaging studies with $\left[{ }^{18} \mathrm{~F}\right] \mathrm{FHBG}$ conducted by Gambhir's group [2]. Our results clearly demonstrate the feasibility of PET imaging with $\left[{ }^{18} \mathrm{~F}\right] \mathrm{FEAU}$ for monitoring the fate of TKGFP expressing progenitor cells proposed in the current application. From the
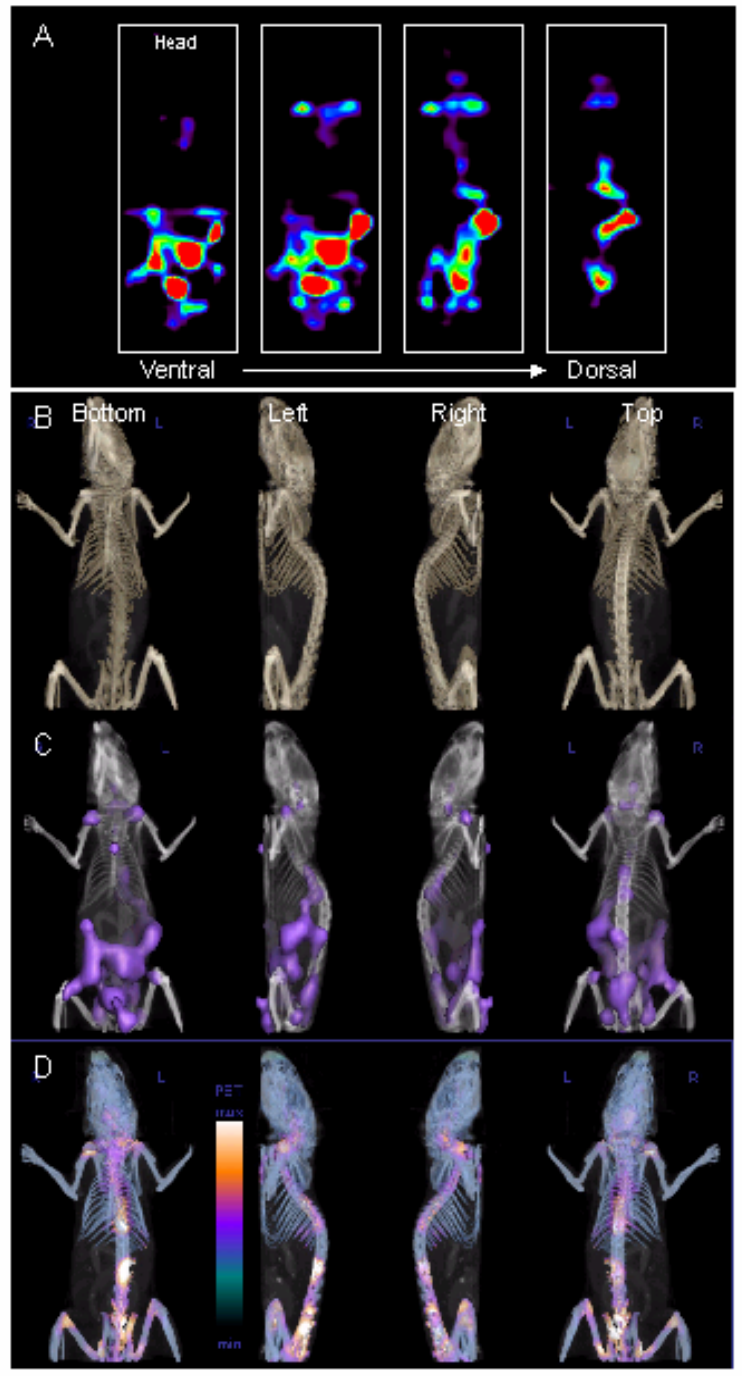

Figure 3. This figure depicts the steps used in fusion of microPET and microCT images. The coronal PET images in parallel planes (A) are co-registered with CT images (B) in space to produce volumetrically fitted PET/CT intermediate images (C). Thereafter, PET signals from spleen (the site of progenitor engraftment), large intestine and bladder (pathways of [18F]FEAU clearance), were digitally removed and the radioactivity localized in bones was color coded to a range of values $(\% \mathrm{ID} / \mathrm{g})$ to produce $\mathrm{PET} / \mathrm{CT}$ parametric images of engraftment into bone structures (D). 
images presented it should be quite obvious to the reader, that PET/CT has sufficient sensitivity and significantly better resolution than $\mathrm{BLI}$, and generates highly accurate and specific functional-anatomical images of progenitor cell trafficking and engraftment (Fig. 3). PET/CT is the leading functional-anatomical imaging modality in clinical radiology today and, therefore, these preliminary results warrant the translation of the proposed imaging approach into clinical studies.

Demonstration of stem cell character of engfrated stem cells. To demonstrate that the engrafted hematopoietic progenitor cells had long-term repopulating characteristics of stem cells, we performed serial bone marrow transplantation procedures at lease in 3 subsequent generations of donor and recipient animals. Additional C57BL/6 (ly5.1+) mice were obtained from The Jackson Laboratory. For Bone marrow transplantation experiments mice were between the ages of 8-12 weeks. Bone marrow cells were removed aseptically from femurs and tibias from two C57BL/6 (ly5.1+) that were pretreated with $5 \mathrm{FU}$ i.p at $150 \mathrm{mg} / \mathrm{kg}$ two days prior to bone marrow harvest. Bone marrow cells were cultivated at $2 \times 10^{\wedge} 6$ cells $/ \mathrm{ml}$ in suspension in stem cell media containing $50 \mathrm{ng} / \mathrm{ml}$ mouse stem cell factor (Sigma), $25 \mathrm{ng} / \mathrm{ml}$ interleukin 6 (Sigma), and $10 \mathrm{ng} / \mathrm{ml}$ interleukin 3 (Sigma) for 48 hours. For the retroviral transduction, $0.5-1.0 \times 10^{\wedge} 6$ cells $/ \mathrm{ml}$ were plated in 6 well plates coated with 4 $\mathrm{ug} / \mathrm{cm}^{\wedge} 2$ of retronectin (Takara). The plates were subsequently blocked with $2 \%$ human sera or BSA for two hours at room temperature. Conditioned cell culture medium collected from transient producer cells containing TGL-retroviral vector was supplemented with $10 \%$ FBS, II-6, II-3 and SCF. After 12 hours the retrovirus containing transfection medium was replaced with new stem cell media. The next day $1 \times 10^{\wedge} 6 \mathrm{BM}$ cells were re-suspended in DMEM and transplanted by tail vein infusion (0.250 ml per mouse). On day 0 prior to transplantation, lethally irradiated recipients received 1100 cGy total body irradiation (137 Cs source) as a split dose with a 3-hour interval between doses (to reduce gastrointestinal toxicity). Prior to imaging, mice were injected intravenously with of $150 \mathrm{mg} / \mathrm{kg}$ D-luciferin in $0.200 \mathrm{ml}$ of PBS and anesthetized for approximately two $\mathrm{min}$ with Isoflurane $(2 \% \mathrm{v} / \mathrm{v})$ prior to being imaged in the supine position. These mice were tracked individually for in vivo luminescence, and subsequently the mice with the greatest signal were

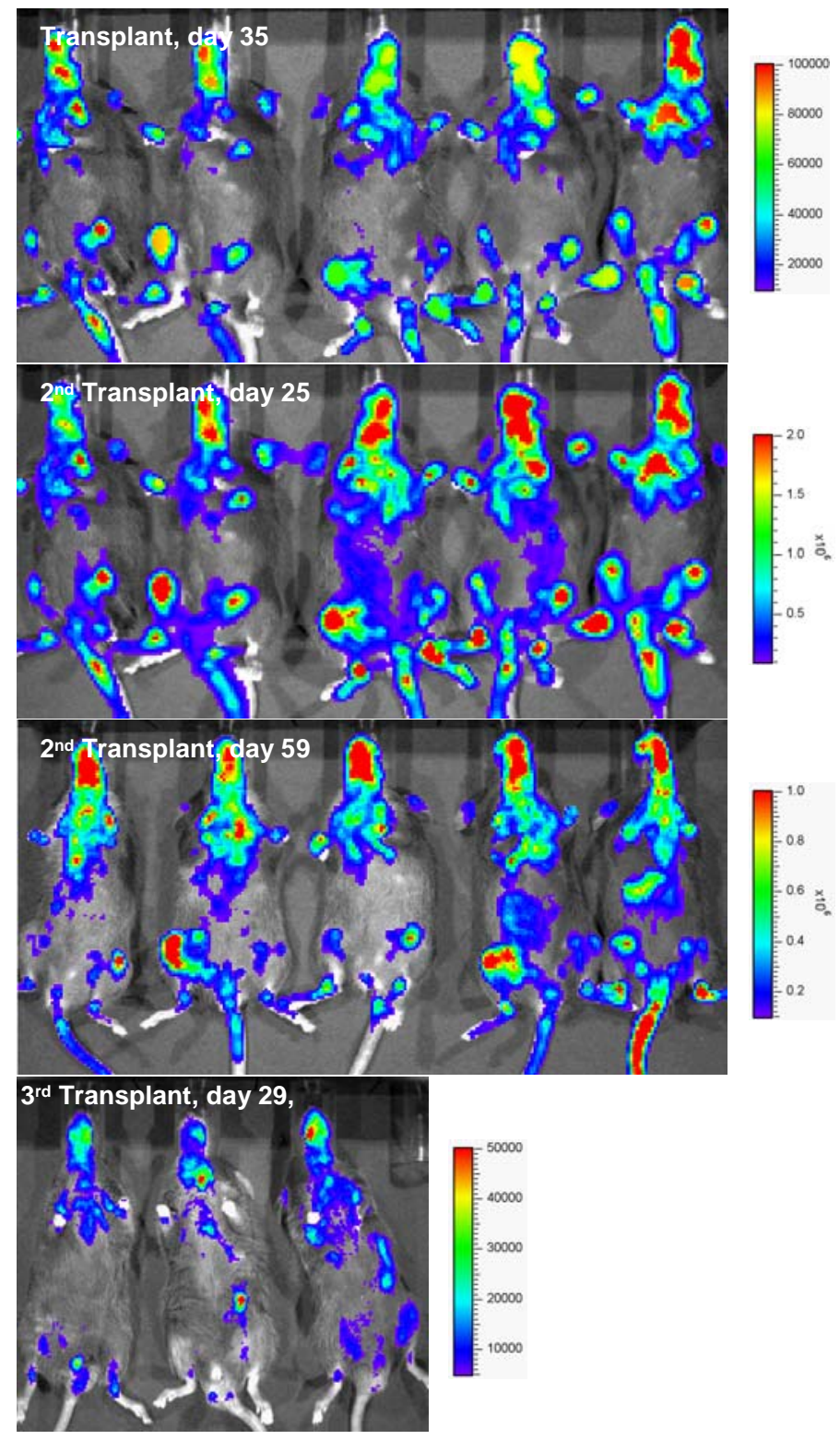

Figure 4. Bioluminescence imaging of bone marrow reconstitution during serial donor-recipient transplantations of TGL-transduced bone marrow-derived progenitors

harvested 81 days later and bone marrow was removed aseptically from femurs and tibias and $10^{\wedge} 7 \mathrm{BM}$ cells were re-suspended in DMEM and injected IV in PBS $0.250 \mathrm{ml}$ per mouse into lethally irradiated C57BL/6 recipients. Mice were imaged as previously described once a week and tracked individually for bioluminescence. Eight weeks later, the bone marrow was harvested as previously described and $4 \times 10^{\wedge} 6$ BM cells were injected intravenously into lethally irradiated and non-irradiated recipients.

The BLI images (Fig. 4) clearly demonstrate that the originally transplanted TGL-transduced bone marrow cells include cells that exhibit properties of long-term repopulating hematopoietic progenitors. 
Dual Reporter Gene Imaging with PET. Previously we developed a new PET reporter gene - reporter probe combination, which is based on the human mitochondrial thymidine kinase type two (hTK2) in which the mitochondrial localization signal locate in the N-terminus of this enzyme was deleted. The fusion of the truncated hTK2 with the monomeric (non-toxic form) of Discosoma coral red fluorescent protein (DsRed) demonstrated the cytoplasmic distribution of this novel fusion reporter enzyme (Fig. 5C). In contrast to it's natural mitochondrial localization, which makes the wildtype hTK2 enzyme metabolically inaccessible and prevents hTK2 from efficiently metabolizing various nucleoside analogues, including FIAU and FEAU (Fig. 5 A-C). In addition, the expression of wild-type hTK2 is usually very low and only increases during mitochondrial division. Truncation of the mitochondrial signal and shift of subcellular localization of mutant hTK2 into cytoplasm resulted in a significantly improved metabolic availability of this enzyme with preservation of it's enzymatic properties for the phosphorylation of thymidine and various nucleoside analogues, including FIAU and FEAU. Interestingly, the hTK2 enzyme exhibited no affinity to acyclic nucleoside analogues, including penciclovir and it's fluorinated analogue $\left[{ }^{18} \mathrm{~F}\right] \mathrm{FHBG}$. For the proof of principle studies, U87 human glioma cells were stably transduced with a newly developed self-inactivating retroviral vector (with deletion in the 3'-LTR) encoding and the TKGFP "sensor" gene driven by the p53-specific promoter and the hTk2RFP "beacon" gene driven by the CMV promoter (p53P in the Fig. 5B). The transduced

TKGFPcmv-TK2RFP obtained using

U87p53sr39 cells were FACS-aided


Figure 5. Development and validation of p53-specific dual PET reporter system. 
selection based on the high levels of hTK2RFP "beacon" expression and low levels of TKGFP "sensor" expression. Under the baseline conditions, the U87p53sr39TKGFP cmvTK2RFP cells exhibited bright cytoplasmic fluorescence and clear (dark) nuclei (Fig. 5C). Exposure of U87p53sr39TKGFPcmvTK2RFP cells to $50 \mu \mathrm{g} / \mathrm{ml}$ of Ethoposide for 2 hours resulted in a rapid development of nuclear expression of TKGFP induced by the activated p53 (Fig. 5D), which was similar to our previous observations [3].

Dual-label radiotracer accumulation studies performed in wild-type U87 cells and U87p53sr39TKGFPcmvTK2RFP in vitro under baseline conditions and after treatment with Etoposide revealed the expected differences in accumulation of $\left[{ }^{14} \mathrm{C}\right] \mathrm{FIAU}$ and $\left[{ }^{3} \mathrm{H}\right] \mathrm{PCV}$ (penciclovir), or $\left[{ }^{14} \mathrm{C}\right] \mathrm{FEAU}$ and $\left[{ }^{3} \mathrm{H}\right] \mathrm{PCV}$ (Fig. 5E). Under the baseline conditions, only insignificant levels of accumulation in wild-type U87 cells were detectable for FIAU and no accumulation was observed with FEAU or PCV. In U87p53sr39TKGFPcmvTK2RFP cells under baseline conditions (high constitutive hTK2RFP and no p53mediated TKGFP expression), the rate of FIAU and FEAU accumulation (expressed in terms of unidirectional flux, Kin) was significantly increased over the baseline, while no accumulation of PCV was detectable. Treatment with Etoposide induced p53 activation and upregulation of sr39TKGFP expression, which in turn mediated a significant increase in FIAU and FEAU accumulation, but most importantly, resulted in high accumulation of PCV (the non-fluorinated analogue of FHBG) (Fig. 5E). The latter confirms the absence of affinity of PCV (and FHBG) to hTK2 and high affinity of PCV to sr39HSV1-TK. At the same time, these results demonstrate a very high affinity of FIAU and FEAU for hTK2 and HSV1-TK enzymes. Subsequent to these studies, we implanted the wild-type U87 and reporter U87p53sr39TKGFPcmvTK2RFP cells into nude mice $\left(10^{6} \mathrm{cells} / \mathrm{site}\right)$ into the opposite shoulders and allowed subcutaneous tumors to develop until about 3-4 $\mathrm{mm}$ in diameter (Fig. 5F). Thereafter, the mice were injected with $\left[{ }^{18} \mathrm{~F}\right] \mathrm{FEAU}(100 \mu \mathrm{Ci} / \mathrm{mouse})$ and imaged on a microPET device (P4 system, Concorde, TN) one hour later. We observed a very clear accumulation of $\left[{ }^{18} \mathrm{~F}\right]$ FEAU in reporter tumors ("beacon" hTK2RFP expression, Fig. 5F-A) and no accumulation in wild-type U87 tumors (the background activity in images has been brought up intentionally to demonstrate that the level of activity in wild-type tumors was indeed no different from the rest of the body). After the accumulated $\left[{ }^{18} \mathrm{~F}\right] \mathrm{FEAU}$ had decayed, on the next day the mice were injected with $\left[{ }^{18} \mathrm{~F}\right] \mathrm{FHBG}$ and imaged with PET again one hour later. The images of $\left[{ }^{18} \mathrm{~F}\right] \mathrm{FHBG}$ obtained under the baseline conditions revealed only very low levels of TKGFP expression (Fig. 5F-B, due to somewhat higher p53-activity in tumors growing in vivo, as was observed before in Doubrovin et at, [3].

When this PET imaging sequence with two radiotracers was repeated in the same mice, but after one treatment with Etoposide (50 mg/kg, i.p.), no difference in $\left[{ }^{18} \mathrm{~F}\right] \mathrm{FEAU}$ accumulation was observed (Fig. 5FC), as compared to baseline levels, which reflects a stable expression levels of the hTK2RFP "beacon" reporter. In contrast, a substantially higher accumulation of $\left[{ }^{18} \mathrm{~F}\right] \mathrm{FHBG}$ was observed (Fig. 5F-D) as compared to that observed during the baseline study, which reflects p53 mediated up-regulation of TKGFP "sensor" reporter expression. In combination with our previous report on successful PET imaging of tumorspecific lymphocyte trafficking and tumor targeting in mice [4] these current preliminary results in transduced tumor xenografts demonstrate the "proof of principle" that the dual-reporter system proposed for the current application on monitoring the trafficking patterns of progenitor cells and their differentiation into various tissues is feasible. For the proposed studies, we will develop self-inactivating retroviral and lentiviral vectors that will carry a similar reporter gene cassette, in which the expression of TKGFP "sensor" reporter will be controlled by a tissue-"specific promoter (e.g., neuro-specific, glial-specific, or endothelium-specific).

1. Cao YA, Wagers AJ, Beilhack A, Dusich J, Bachmann MH, Negrin RS, Weissman IL, Contag CH. Shifting foci of hematopoiesis during reconstitution from single stem cells. Proc Natl Acad Sci U S A. 2004 Jan 6;101(1):221-6.

2. Su, et al. Molecular Imaging and Biology, 2004 In Press.

3. Doubrovin M, Ponomarev V, Beresten T, Balatoni J, Bornmann W, Finn R, Humm J, Larson S, Sadelain M, Blasberg R, Gelovani Tjuvajev J. Imaging transcriptional regulation of p53-dependent genes with positron emission tomography in vivo. Proc Natl Acad Sci U S A. 2001 Jul 31;98(16):9300-5.

4. Koehne G, Doubrovin M, Doubrovina E, Zanzonico P, Gallardo HF, Ivanova A, Balatoni J, Teruya-Feldstein J, Heller G, May C, Ponomarev V, Ruan S, Finn R, Blasberg RG, Bornmann W, Riviere I, Sadelain M, O'Reilly RJ, Larson SM, Tjuvajev JG. Serial in vivo imaging of the targeted migration of human HSV-TK-transduced antigenspecific lymphocytes. Nat Biotechnol. 2003 Apr;21(4):405-13. 


\section{Commitment of neural progenitor cells to a neuronal fate}

Commitment of progenitor cells in the nervous system to a neuronal fate is regulated by basic helix-loophelix (bHLH) transcription factors, such as Mash1, Math3, Neurogenin, and NeuroD. Neurogenin, initiates stem cell differentiation into a neuronal phenotype by binding to the CBP/p300/Smad1 nuclear complex, which activates the expression of downstream nuclear differentiation factors such as NeuroD. Neurogenin also acts to prevent gliogenesis by diverting the binding of the CBP/p300/Smad1 to glial-specific promoters thereby sequestering gliogenesis. NeuroD is transiently expressed in central and peripheral nervous system during terminal differentiation of progenitor cells into neurons.

The terminal stage of neuronal differentiation involves the expression of neuron-specific functional and structural genes such as SCG10, sodium channel type II, synapsin, and glutamate receptor. The expression of these terminal differentiation genes is maintained in a suppressed state by the neuron-restrictive silencing factor (NRSF)/repressor element-1 (RE1) silencing transcription factor (REST). Thus, terminal differentiation of stem cells into neurons requires the de-repression of neuronal promoters containing neuron-restrictive silencing elements (NRSE)/Repressor element 1 (RE1).

Neuron-specific gene expression in mesenchymal stem cells demonstrates their potential for neuronal differentiation. Differentiation of MS cells into neurons by chemical and genetic means has been described. Preliminary assessment of gene expression demonstrates that MS cells express a wide variety of neuron-specific genes associated with early and intermediate stages of neuronal differentiation. To determine if commitment to a neuronal fate is stimulated by NGF in MS cells, a comparative study was conducted using an established neuronal differentiation model. Rat pheochromocytoma (PC-12) cells undergo neuronal differentiation within 24 hours following NGF treatment and exhibit characteristic features of differentiation, namely neurite outgrowth. PC-12 and MS cells were plated and treated in parallel with NGF (50 ng/mL). The expression of several early, intermediate, and late differentiation markers was then determined by reverse transcriptase-polymerase chain reaction (RT-PCR) analysis 48 hours following treatment.

Undifferentiated MS cells constitutively express a variety of neuron-specific genes associated with early and intermediate neuronal differentiation. As shown in Figure 1, PC-12 cells exhibited high levels of NGF receptor (NGFR) expression relative to the expression of glyceraldehyde phosphate dehydrogenase (GAPDH) internal standard. MS cells exhibited lower levels of NGFR expression relative to GAPDH. Nonetheless, responsiveness of MS cells to nerve growth factor (NGF) is consistent with the, albeit low, expression of NGFR. The baseline levels of NGFR expression were not affected by NGF treatment in either cell line.

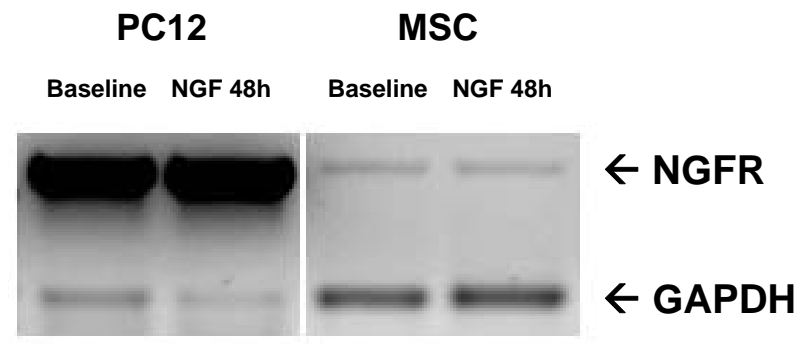

Figure 1. Expression of NGFR in PC12 cells and Rat MS cells. PC12 and MS cells were plated in 100 mm dishes and treated with NGF $(50 \mathrm{ng} / \mathrm{mL})$ for 48 hours. RT-PCR analysis demonstrated significant levels of NGFR expression in PC12 cells relative to the GAPDH internal standard. MS cells also exhibited NGFR expression, albeit at levels lower than the GAPDH standard. Compared to baseline levels, the expression of NGFR was not affected by NGF treatment in both cell lines.

Early neurogenic events are initiated by bHLH transcription factors (Mash1, Math3, Neurogenin, NeuroD). Thus, expression of these factors would indicate a cellular commitment towards a neuronal lineage. In PC12 cells, neurogenin expression was not detected 48 hours following NGF treatment, whereas MS cells exhibited an apparent induction of neurogenin expression (Figure 2). NeuroD expression was detected in PC12 cells, but the levels following NGF treatment were relatively similar to baseline. In contrast, MS cells exhibited an NGF-induced elevation of NeuroD expression. Since neurogenin and NeuroD levels were 
measured 48 hours post-treatment, an elevation followed by a subsequent drop in the expression of the transcription factors is a plausible event given the higher level of NGFR expression in PC-12 cells. Assessment of expression at earlier time points, within a few hours following treatment, is necessary to determine if any increases in neurogenin or NeuroD occur in PC-12 cells. The detectable increases of neurogenin and NeuroD levels in MS cells at 48 hours may reflect a delayed response to NGF due to the significantly lower levels of NGFR.

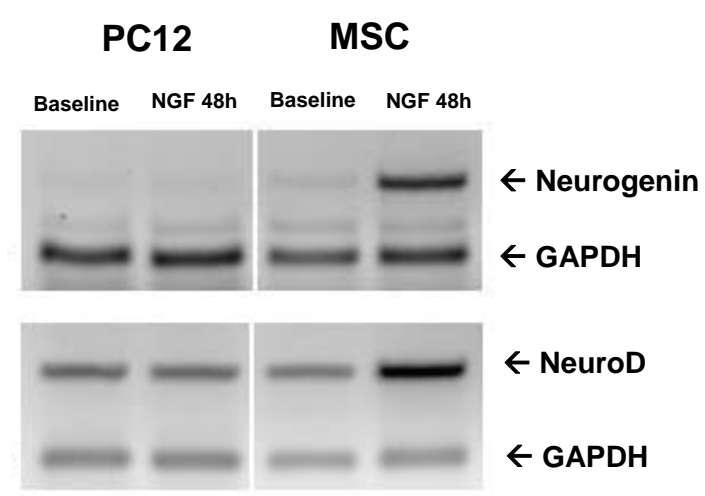

Figure 2. Expression of neurogenin and NeuroD in PC-12 and Rat MS cells. Neurgenin was not detected in PC-12 48 hours following treatment with NGF $(50 \mathrm{ng} / \mathrm{mL})$. NeuroD was detected but NGF did not appear to influence the levels of expression at 48 hours. The levels of neurogenin and NeuroD were, however, measurably elevated in response to NGF.

Nestin is an early indicator on neuronal differentiation expressed in neural progenitor cells. NGF-induced elevation of nestin was observed in PC-12 and MS cells indicating a directional commitment of both cell types towards a neuronal fate (Figure 3 ).

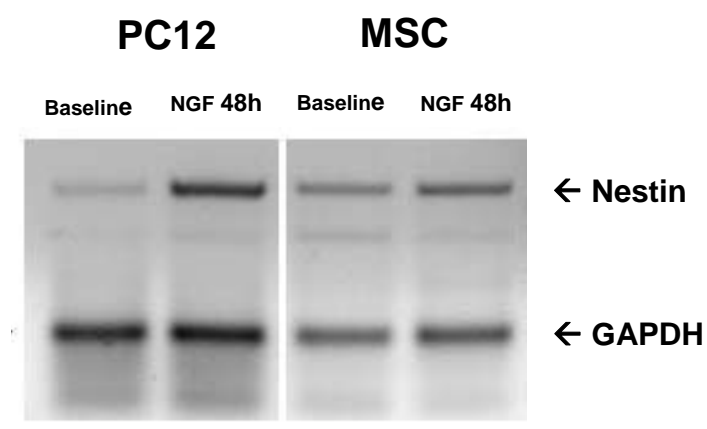

Figure 3. Expression of the early differentiation factor nestin. An elevation of nestin expression was observed in PC-12 and MS cells indicating an NGF-induced cellular commitment towards a neuronal lineage.

Terminal differentiation is associated with the expression of structural proteins, where physical, morphological, and functional changes become apparent. In neurons, such changes are most obviously manifested in the form of neurite outgrowth. Thus, expression of genes associated with axonal growth and maintenance was evaluated in PC-12 and MS cells. As illustrated in Figure 4, the expression of growthassociated protein-43 (GAP-43), neurofilament-M, and $\beta$-tubulin III was inducible in PC-12 cells but not in MS cells. This observation correlates with the appearance of neurite outgrowth in PC-12 cells (Figure 5b) and the lack of neuron-like morphological changes in MS cells (Figure $5 \mathrm{~d}$ ). Doublecortin and microtubuleassociated protein-1B (MAP-1B), in contrast, were expressed constitutively in both cell lines. 


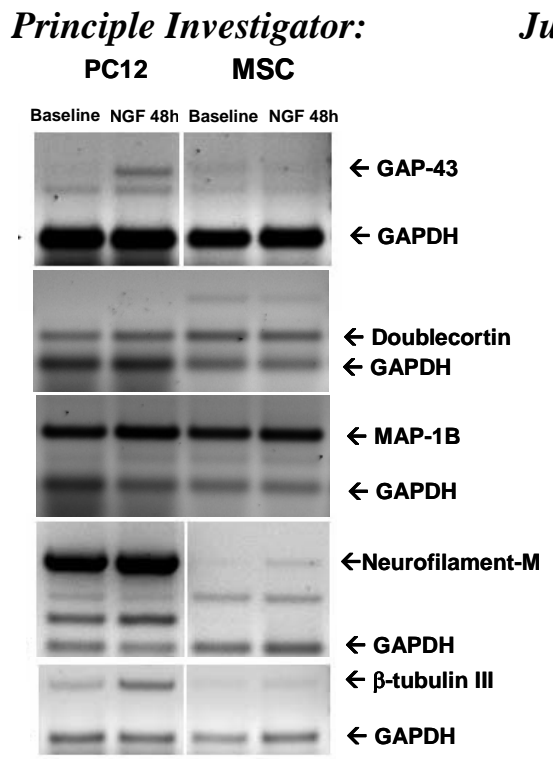

Figure 4. Expression of neuronal terminal differentiation markers. Expression of GAP-43, Neurofilament-M, and $\beta$ tubulin III was inducible in PC-12 cells and correlated with presence of neurite outgrowth.
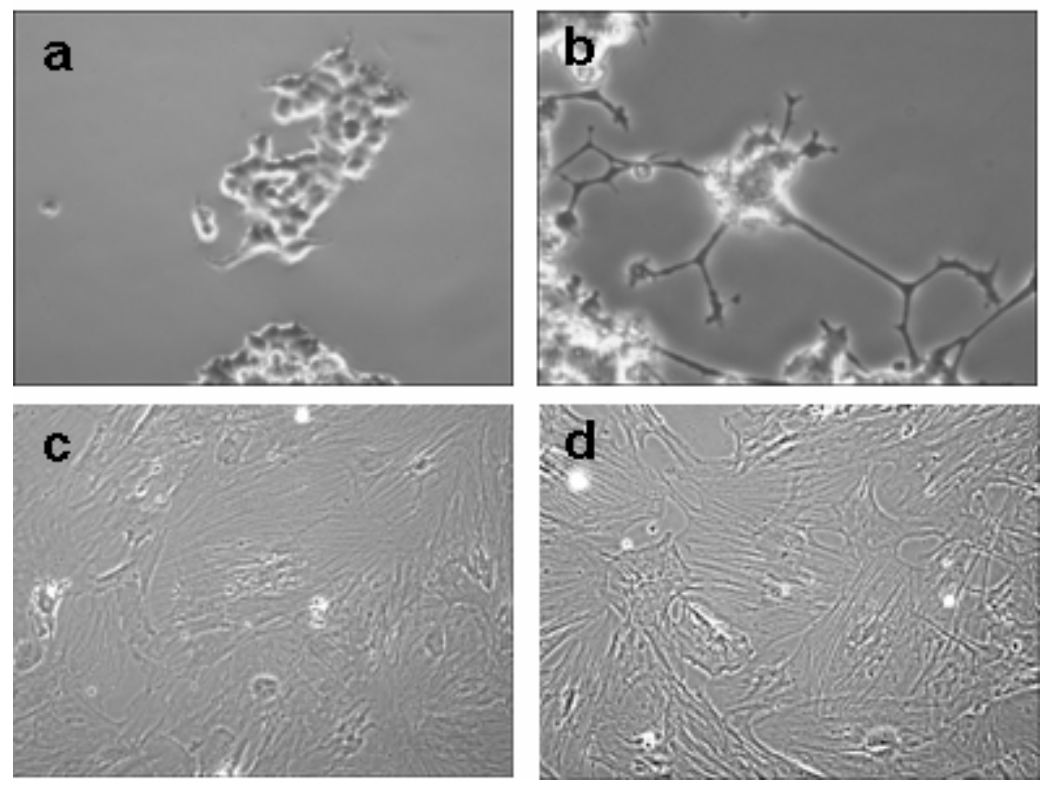

Figure 5. Cellular morphology of PC-12 and MS cells. Untreated PC-12 cells (a) exhibit neurite outgrowth 48 hours following NGF (50 ng/mL) treatment (b). Untreated (c) and NGF-treated (d) MS cells do not exhibit morphological changes.

Although MS cells do not exhibit morphological changes upon treatment with NGF, their potential for neuronal differentiation is demonstrated by the expression of neuron-specific genes such as neurogenin, NeuroD, nestin, doublecortin, and MAP-1B. Moreover, the lack of terminal differentiation may potentially be due to desensitization to NGF by inactivation of the already low levels of NGFR expressed in MS cells. Therefore, the approach for terminal neuronal differentiation of MS cells will be based on stable transfection of the early transcription factors neurogenin and NeuroD. Prolonged expression neurogenin or NeuroD is expected to advance MS cells into the final stages of differentiation.

The gene expression profile provides insight into the degree of progression of the neuronal differentiation pathway in MS cells. MS cells appear poised to enter the initial stages of differentiation as indicated by the expression of nestin. The inducible expression of the bHLH factors neurogenin and NeuroD indicates the entry of MS cells into the intermediate stage of differentiation. However, the lack of $\beta$-tubulin III and GAP-43 expression demonstrates a lack of terminal progression of the neuronal differentiation pathway in MS cells. This fact correlates with presence of the NRSE/REST sequence in the $\beta$-tubulin III promoter. The 
NRSE/REST sequences are de-repressed during terminal differentiation leading to the expression of genes required by functional proteins such as sodium channel type II, synapsin, glutamate receptor, and the acetylcholine receptor.

Design of reporter gene vector for the determination of stem cell viability and differentiation following engraftment. Preclinical assessment of the viability and state of differentiation will be determined by repetitive micro-PET imaging of MS cells transduced with a retroviral dual reporter system. Cellular viability will be monitored using the HSV1-thymindine kinase "beacon" which will be constitutively expressed. Terminal differentiation will be imaged through the expression of mitochondrial human thymidine kinase 2 under the control of a neuron-specific promoter expressed only in terminally differentiated cells.

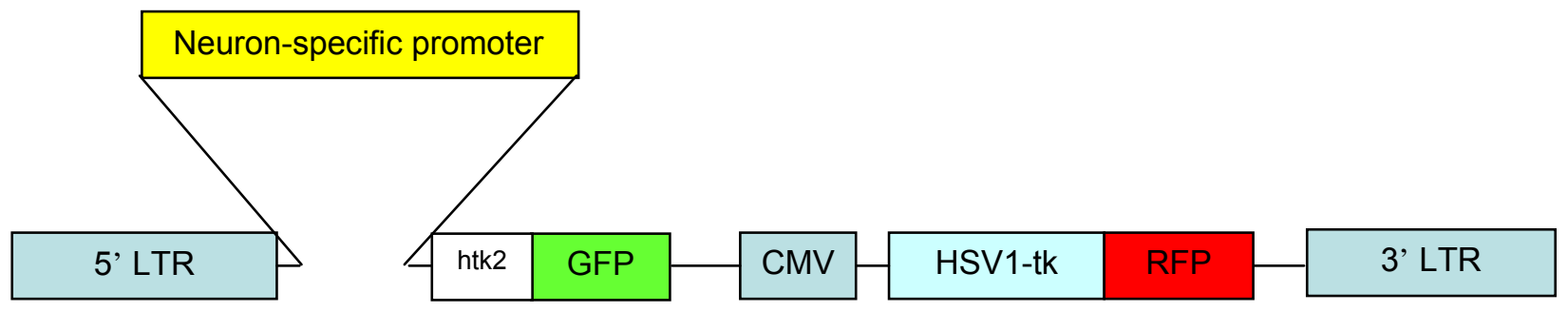

\section{Differentiation signal}

Beacon

The initial dual reporter construct produced utilized the neuron-specific enolase promoter to drive the expression of htk2-GFP. However, transfection of MS cells revealed that the promoter was not a valid indicator of neuronal differentiation since both the "beacon" and "differentiation" signals were both constitutively expressed (Figure 6). RT-PCR analysis confirmed the microscopic observation by showing that NSE expression was constitutive in untreated and NGF-treated PC-12 and MS cells. Current efforts are underway to construct a new reporter system based on the use of the synaptosin promoter, which contains a NRSE/REST sequence approximately 400 bp upstream of the transcriptional start site. Thus, this promoter will only become activated during the terminal differentiation stages.

\section{PC12 MSC}

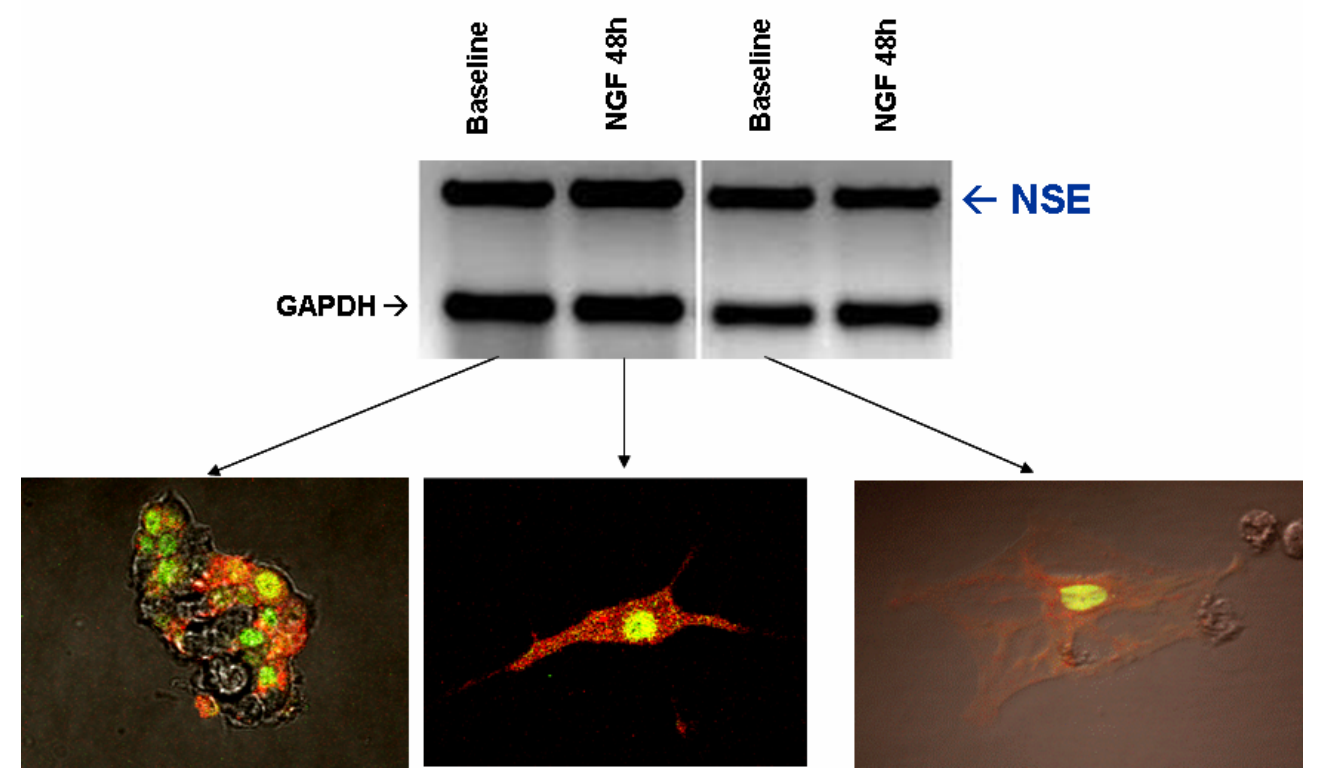

Figure 6. Constitutive expression of NSE in PC-12 and MS cells. The cells were transduced with a dual reporter vector expressing an htk2-GFP fusion protein under the control of the NSE promoter and an HSV1-tk-RFP "beacon" reporter under the control of CMV. The NSE promoter was actively expressed in untreated and NGF-treated cells as indicated by the presence green fluorescence in the nucleus. RT-PCR analysis revealed that NSE is constitutively expressed in undifferentiated cells and therefore cannot serve as a valid indicator of differentiation. 


\section{REFERENCES.}

1. Lee Reinhardt $R$, et al., (2001) Nature 410, 101-105.

2. Atkins RC, Ford WL. (1975) J Exp Med. 141(3):664-80

3. Constantin G, et al., (1997) J Immunol Methods 203(1):35-44

4. Hellerstein M, et al., (1999) Nat Med; 5(1):83-89.

5. Gobuty AH, et al., (1977) J Nucl Med. Feb;18(2):141-146.

6. Papierniak CK, et al., (1976) J Nucl Med.;17(11):988-992.

7. Korf J, et al., (1998);J Nucl Med. 39(5):836-841.

8. Rannie, G.H., et al., (1977) Clin. Exp. Immunol. 29(3): 509-514.

9. Adonai, N., et al., (2002) Proc. Natl. Acad. Sci. U S A 99(5): 3030-3035.

10. Kasi LP, et al., (1995) Int J Gynecol Cancer 5(3):226-232.

11. Dillman RO, et al., (1997) Cancer Biother Radiopharm;12(2):65-71.

12. Schelper, R.L., \& Adrian, E.K. Jr. (1986) J. Neuropathol. Exp. Neurol; 45(1), 1-19.

13. Tjuvajev JG, et al., (1994) J Nucl Med; 35(9):1407-1417.

14. Blasberg RG, et al., (2000) Cancer Res; 60(3):624-635.

15. Choi, S., et al., (1997) Int. J. Imaging Sci. Technol; 8: 263-276.

16. Jacobs, R.E., et al., (1999) Trends Cell Biol; 9:73-76.

17. Johnson, G.A. et al. (1993).,Magn. Reson. Q; 9:1-30.

18. Safarik, I. \& Safarikova, M.. (1999) J Chromatogr; 722, 33-53.

19. Weissleder, R., et al., (1997). J. Magn. Reson. Imaging 7, 258-263.

20. Schoepf, U., et al., (1998) BioTechniques 24, 642-651.

21. Dodd, S.J. et al. (1999)., Biophys. J. 76, 103-109.

22. Hawrylak, N. et al. (1993) Exp. Neurol. 121, 181-192.

23. Bulte, J., et al., (1999) Radiol. Soc. North Am. 213, 225.

24. Weissleder, R. et al. (2000).,Nat. Med. 6, 351-354.

25. Schwarze, S.R., et al., (1999) Science 285, 1569-1572.

26. Fawell, S. et al. (1994)., Proc. Natl. Acad. Sci. USA 91, 664-668.

27. Nagahara, H. et al. (1998), Nat. Med. 4, 1449-1452.

28. Lewin M., et al., (2000) Nat Biotech 18(4):410-414.

29. Rooney CM, et al. (1995) Lancet 345:9-13.

30. Verzeletti S, et al.,(1998) Hum Gene The; 9(15):2243-2251.

31. Bonini C, et al., (1997 Science; 276(5319):1719-1724.

32. Richardson C, Bank A., (1996) Mol Cell Biol; 16(8):4240-4247.

33. Cui Y, et al., (2002) Blood; 99(2):399-408.

34. Hardy J, et al., (2001). Exp Hematol 29, 1353-1360.

35. Tjuvajev JG, et al., (1995) Cancer Res;55(24):6126-6132.

36. Gambhir SS, et al., (2000) Neoplasia 2(1-2):118-138.

37. Banerjee D. (1999) Curr Opin Mol Ther 1(4):517-520.

38. Floeth FW, et al., (2001) Cancer Gene Ther 8(11):843-851.

39. Jacobs A, et al., (1999) Neoplasia;1(2):154-161.

40. Doubrovin M., et al., (2001) Proc Natl Acad Sci U S A ;98(16):9300-9305.

41. Ponomarev V, et al., (2001) Neoplasia; 3(6):480-488.

42. Jacobs A, et al., (2001) Lancet 358(9283):727-729.

43. Yaghoubi S., et al., (2001) J Nucl Med; 42(8):1225-1234.

44. Liang Q., et al., (2001) Gene Ther; 8(19): 1490-1498.

45. Satyamurthy N., et al., (1990) Int J Rad Appl Instrum [A]; 41(2):113-129.

46. Ferrara JL, et al., (1999) Biol Blood Marrow Transplant; 5:347-356.

47. Folkman, J. Tumor angiogenesis: therapeutic implications. N Engl J Med 1971; 285:1182-6.

48. Holash, J., et al. Science 1999;284:1994-8.

49. Carmeliet, P. and Jain, R. Nature 2000; 407:249-57.

50. De Palma, et al. Nat Med 2003; 9:789-95.

51. Prockop, D. J. Science 1997, 276:71-4.

52. Pittenger, M. F., et al. Science 1999;284:143-7. 
53. Bruder, S. P., et al. J Cell Biochem 1994;56:283-94.

54. Jin, H. K., et al. J Clin Invest 2002;109:1183-91.

55. Spees, J. L., et al. Proc Natl Acad Sci U S A 2003;100:2397-402.

56. Reyes, M., et al. J Clin Invest 2002;109:337-346.

57. Orlic, D., et al. Nature 2001;410:701-5.

58. Studeny, M., et al. Cancer Res 2002;62:3603-8.

59. Nakamura, K., et al. Gene Ther 2004;11;1155-64.

60. Doubrovin, M., et al. Proc Natl Acad Sci U S A 2001;98:9300-5.

61. Jacobs, A., et al. Neoplasia 1999;1:154-61.

62. Ray, P., et al. Cancer Res 2003;63:1160-5.

63. Ray, P., et al. Cancer Res 2004;64:1323-30.

64. Chen, W. S., et al. Int J Cancer 2001;91:894-9.

65. Hung, S. C., et al. Int J Cancer 2004;110:313-9.

66. Chang, L. J. \& Zaiss, A. K. (2002) Methods Mol Med 69, 303-18.

67. Tjuvajev, J. G., et al. J Nucl Med 2002;43:1072-83.

68. Deng, W. P., et al. Eur J Nucl Med Mol Imaging 2004;31:99-109.

69. Fareed, M. U. \& Moolten, F. L. Gene Ther 2002;9:955-62.

70. Shiue, G. G., et al. Nucl Med Biol 2002;28:875-83.

71. Lee, K., et al. Phys Med Biol 2004;49:4563-78.

72. Jockusch, H., et al. J Histochem Cytochem 2003;51:401-4.

73. St Croix, B., et al. Science 2000;289:1197-202.

74. Hung, S. C., et al. Stem Cells 2002;20:249-58.

75. Miyagawa, M., et al. J Nucl Med 2004;45:1917-23.

76. Wu, J. C., et al. Circulation 2003;108:1302-5.

77. Dubey, P., et al. Proc Natl Acad Sci U S A 2003;100:1232-7.

78. Koehne, G., et al. Nat Biotechnol 2003;21:405-13.

79. Aboody, K. S., et al. Proc Natl Acad Sci U S A 2000;97:12846-51.

80. Relf, M., et al. Cancer Res 1997;57:963-9.

81. Cottler-Fox, M. H., et al. (2003) Hematology (Am Soc Hematol Educ Program), 419-37.

82. Hung, S. C., et al. Stem Cells 2002;20:522-9.

83. Duda, D. G., et al. Cancer Res 2004;64:5920-4.

84. Alauddin, M. M., et al. J Nucl Med 2004;45:2063-9.

85. Serganova, I., et al. Cancer Res 2004;64:6101-8.

86. Tjuvajev, J. G., et al. Cancer Res 1998;58:4333-41.

87. Wen, B., et al. Eur J Nucl Med Mol Imaging 2004;31:1530-8. 